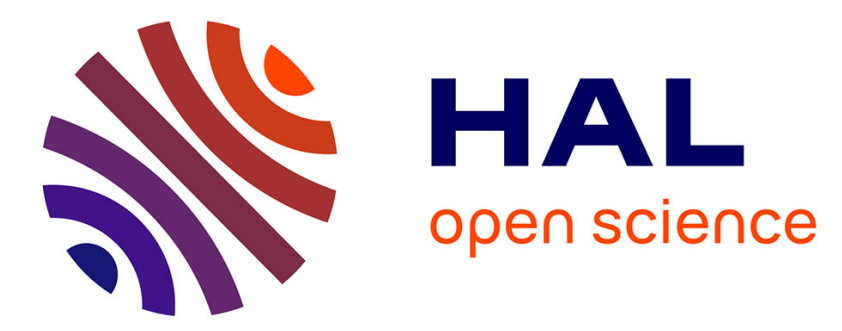

\title{
Synthesis, Characterization and Encapsulation \\ Properties of Rigid and Flexible Porphyrin Cages Assembled from N-Heterocyclic Carbene-Metal Bonds
}

Ludivine Poyac, Clémence Rose, Mohammad Wahiduzzaman, Aurélien

Lebrun, Guillaume Cazals, Charles H. Devillers, Pascal G Yot, Sébastien

Clément, Sébastien Richeter

\section{To cite this version:}

Ludivine Poyac, Clémence Rose, Mohammad Wahiduzzaman, Aurélien Lebrun, Guillaume Cazals, et al.. Synthesis, Characterization and Encapsulation Properties of Rigid and Flexible Porphyrin Cages Assembled from N-Heterocyclic Carbene-Metal Bonds. Inorganic Chemistry, 2021, 60 (24), pp.19009-19021. 10.1021/acs.inorgchem.1c02868 . hal-03518954

\section{HAL Id: hal-03518954 \\ https://hal.science/hal-03518954}

Submitted on 10 Jan 2022

HAL is a multi-disciplinary open access archive for the deposit and dissemination of scientific research documents, whether they are published or not. The documents may come from teaching and research institutions in France or abroad, or from public or private research centers.
L'archive ouverte pluridisciplinaire HAL, est destinée au dépôt et à la diffusion de documents scientifiques de niveau recherche, publiés ou non, émanant des établissements d'enseignement et de recherche français ou étrangers, des laboratoires publics ou privés. 


\section{Synthesis, Characterization and Encapsulation}

\section{Properties of Rigid and Flexible Porphyrin Cages}

\section{Assembled from N-Heterocyclic Carbene-Metal}

\section{Bonds}

Ludivine Poyac, $\|,[\mathrm{a}]$ Clémence Rose, $\|,{ }^{[\mathrm{a}]}$ Mohammad Wahiduzzaman, ${ }^{[\mathrm{a}]}$ Aurélien Lebrun, ${ }^{[\mathrm{b}]}$

Guillaume Cazals, ${ }^{[\mathrm{b}]}$ Charles H. Devillers, ${ }^{[\mathrm{c}]}$ Pascal G. Yot, ${ }^{[\mathrm{a}]}$ Sébastien Clément, ${ }^{[\mathrm{a}]}$ and

Sébastien Richeter* ${ }^{[\mathrm{a}]}$

Corresponding Author:

Sébastien Richeter - ICGM, Univ Montpellier, CNRS, ENSCM, Montpellier 34293, France;

Email: sebastien.richeter@umontpellier.fr

Affiliations :

[a] ICGM, Univ Montpellier, CNRS, ENSCM, Montpellier 34293, France

[b] LMP, Université de Montpellier, Montpellier 34293, France 
[c] ICMUB UMR6302, CNRS, Univ. Bourgogne Franche-Comté, 9 avenue Alain Savary, Dijon 21078, France

KEYWORDS. porphyrinoids ; carbene ligands ; cage compounds ; self-assembly ; host-guest systems.

ABSTRACT : Four porphyrins equipped with imidazolium rings on the para positions of their meso aryl groups were prepared and used as tetrakis(N-heterocyclic carbene) (NHC) precursors for the synthesis of porphyrin cages assembled from eight NHC-M bonds $\left(\mathrm{M}=\mathrm{Ag}^{+}\right.$or $\left.\mathrm{Au}^{+}\right)$. The conformation of the obtained porphyrin cages in solution and their encapsulation properties strongly depend on the structure of the spacer $-\left(\mathrm{CH}_{2}\right)_{\mathrm{n}^{-}}(\mathrm{n}=0$ or 1$)$ between meso aryl groups and peripheral NHC ligands. In absence of methylene groups $(n=0)$, porphyrin cages are rather rigid and the short porphyrin-porphyrin distance prevents encapsulation of guest molecules like 1,4diazabicyclo[2.2.2] octane (DABCO). By contrast, the presence of methylene functions $(n=1)$ between meso aryl groups and peripheral NHCs offers additional flexibility to the system allowing the inner space between the two porphyrins to expand enough to encapsulate guest molecules like water molecules or DABCO. The peripheral NHC-wingtip groups also play a significant role on the encapsulation properties of the porphyrin cages. 


\section{INTRODUCTION}

Coordination driven self-assembly of supramolecular complexes is an active field of research to devise original scaffolds with different sizes and shapes. ${ }^{1,2}$ Many fascinating supramolecular architectures built upon the formation of metal-ligand bonds were reported in the literature like helicates ${ }^{3,4}$ and knots..$^{5-7}$ Metallocages are supramolecular complexes possessing confined nanospaces that may be used for host-guest chemistry ${ }^{8,9}$ and several applications ranging from the encapsulation of molecular species for sensing ${ }^{10}$ or catalysis ${ }^{11-15}$ to the design of drug delivery systems for biomedical applications. ${ }^{16-19}$ Incorporating $\pi$-conjugated systems into the ligands structure can bring several advantages such as structural rigidity and optoelectronic properties. In this context, porphyrins are particularly appealing molecular scaffolds to design innovative metallocages. ${ }^{20}$ Obviously, porphyrins with their large structure and their $D_{4 h}$ symmetry are attractive building blocks to design metallocages with expanded three-dimensional cavities which are suitable for guest encapsulation ${ }^{21-25}$ and catalysis. ${ }^{26-28}$ Porphyrins are also stable photoand electroactive $\pi$-conjugated systems and there are well-established synthetic procedures to functionalize their meso and $\beta$-pyrrolic positions. ${ }^{29}$ These macrocycles can also incorporate metal ions into their cavity, which can coordinate different ligands. This strategy has been exploited by Osuka's group to synthesize metallocages containing several porphyrins ${ }^{30}$ Cage-like structures containing two porphyrins assembled face-to-face, namely cofacial porphyrin dimers, are particularly investigated since they can be used as model compounds for photoinduced energy/electron transfer processes between a donor and an acceptor. ${ }^{31-33}$ Cofacial porphyrin dimers are also suitable structures to investigate encapsulation processes. ${ }^{20,34-36}$ In this case, porphyrins play a key role in the encapsulation process since their large $\pi$-delocalized systems and their inner metal centers can stabilize aromatic guest molecules inside the cavity through $\pi$ - $\pi$-stacking 
interactions or suitable ligands through the formation of coordination bonds. For these reasons, cofacial porphyrin dimers are particularly investigated in catalysis, notably for small molecule activation reactions like $\mathrm{O}_{2}$ reduction reactions $(\mathrm{ORR})^{37,38}$ and $\mathrm{CO}_{2}$ reduction reactions. ${ }^{39}$

Compared to systems built upon the formation of covalent bonds, the synthesis of cofacial porphyrin dimers through self-assembly processes is appealing because the yields are usually higher and purification procedures are easier. Most of coordination driven self-assembled cofacial porphyrin dimers are Werner-type coordination compounds built upon formation of metal-ligand bonds with nitrogen, oxygen, sulfur or phosphorus donor atoms of polydentate ligands. Some of them were proven to be efficient for ORR. ${ }^{40,41}$ Recently, we reported the synthesis of the first cofacial porphyrin dimers assembled from eight N-heterocyclic carbene-metal (NHC-M) bonds. ${ }^{42}$ The synthetic strategy consists in the self-assembly of the two porphyrins through labile NHC- $\mathrm{Ag}^{+}$ bonds followed by the replacement of $\mathrm{Ag}^{+}$by $\mathrm{Au}^{+}$(transmetalation reaction). This two-step procedure allowed us to easily obtain very stable porphyrin cages, although an initial self-assembly process was used for their synthesis. Inspired by these preliminary findings, we describe here the synthesis and characterization of novel porphyrin cages assembled from NHC-M bonds $\left(\mathrm{M}=\mathrm{Ag}^{+}\right.$ or $\mathrm{Au}^{+}$). In this study, two types of porphyrins equipped with imidazolium rings on the para positions of their meso aryl groups were used as tetrakis(NHC) precursors. They differ by the nature of the spacer $-\left(\mathrm{CH}_{2}\right)_{\mathrm{n}^{-}}(\mathrm{n}=0$ or 1) between the meso aryl groups and the peripheral NHCs. In absence of methylene group $(\mathrm{n}=0)$, porphyrin-based tetrakis(NHC) are rather rigid, while the presence of methylene functions $(\mathrm{n}=1)$ between the meso aryl groups and the peripheral NHCs brings additional flexibility. The aim of this work is to investigate the impact of the introduction of these methylene groups (i) on the conformation of the obtained porphyrin cages in solution, and (ii) on their encapsulation properties. Indeed, the flexibility offered by the $s p^{3}$ carbon atoms of the 
methylene groups allows the two porphyrins of the cages to move away from each other enabling guest molecules to be encapsulated if the cavity is large enough. The role played by the peripheral NHC-wingtip groups on the conformation of the obtained porphyrin cages and their encapsulation properties is also investigated.

\section{EXPERIMENTAL SECTION}

Materials. Reactions needing inert atmosphere were performed under argon using ovendried glassware and Schlenk techniques. All solvents were obtained from commercial suppliers and used as received. Dry acetonitrile and DMSO were purchased from Alfa Aesar. DMF was purchased from Sigma-Aldrich. Dry $\mathrm{CH}_{2} \mathrm{Cl}_{2}$ and THF were obtained by a PureSolve MD5 solvent purification system from Innovative Technology. Silver(I) oxide 99\% $\left(\mathrm{Ag}_{2} \mathrm{O}\right)$ was purchased from Fluorochem and gold(I) complex $[\mathrm{AuCl}(\mathrm{tht})](\mathrm{tht}=$ tetrahydrothiophene) was prepared according

to the procedure described in the literature. ${ }^{43}$ Pyrrole $(>99 \%)$ was purchased from TCI and distilled under reduced pressure before use. Imidazole (99.5\%), 4-fluorobenzaldehyde (98\%), iodomethane (99\%), potassium hexafluorophosphate $(>99 \%)$ and zinc(II) acetate dihydrate (>98\%) were purchased from Sigma-Aldrich. Hydrogen tetrachloroaurate(III) trihydrate, ACS 99.99\% (metals basis), $\mathrm{Au} 49.0 \%$ min was used as starting material and purchased from Alfa Aesar. Porphyrins $2 \mathbf{a}$ and $\mathbf{2} \mathbf{c}$ were prepared according to the procedures described in the literature. ${ }^{44,45}$ Porphyrins $4 \mathbf{a}-\mathbf{d}$ were prepared according to procedures described in the Supporting Information. TLC were carried out on Merck DC Kieselgel 60 F-254 aluminium sheets and spots were visualized with UV-lamp $(\lambda=254 / 365 \mathrm{~nm})$ if necessary. Preparative purifications were performed by silica gel column chromatography (Merck 40-60 $\mu \mathrm{M})$. 
Instruments and methods. NMR spectroscopy and MS spectrometry were performed at the Laboratoire de Mesures Physiques (LMP) of the University of Montpellier (UM). ${ }^{1} \mathrm{H}, \mathrm{COSY}$, ROESY, HSQC, HMBC, ${ }^{13} \mathrm{C}\left\{{ }^{1} \mathrm{H}\right\},{ }^{31} \mathrm{P}\left\{{ }^{1} \mathrm{H}\right\},{ }^{19} \mathrm{~F}\left\{{ }^{1} \mathrm{H}\right\}$ and DOSY NMR spectra were recorded on Bruker $400 \mathrm{MHz}$ Avance III HD, $500 \mathrm{MHz}$ Avance III or $600 \mathrm{MHz}$ Avance III spectrometers at 298 K. Deuterated solvents DMSO- $d_{6}, \mathrm{CD}_{3} \mathrm{CN}$ and $\mathrm{CD}_{2} \mathrm{Cl}_{2}$ were used as received (purchased from Sigma-Aldrich). ${ }^{1} \mathrm{H}$ and ${ }^{13} \mathrm{C}\left\{{ }^{1} \mathrm{H}\right\}$ NMR spectra were calibrated to TMS on the basis of the relative chemical shift of the residual non-deuterated solvent as an internal standard. Chemical shifts $(\delta)$ are expressed in ppm and coupling constants values $\left({ }^{\mathrm{n}} J\right)$ are expressed in Hz. Abbreviations used for NMR spectra are as follows: s, singlet; d, doublet; t, triplet; quint, quintuplet; sext, sextuplet; sept, septuplet; m, multiplet; br, broad. No satisfactory elemental analysis data were obtained for porphyrin cages. High Resolution Mass spectra (HRMS) were recorded on a Bruker MicroTof QII instrument in positive/negative modes (ESI). UV-Visible absorption spectra were recorded in $\mathrm{CH}_{2} \mathrm{Cl}_{2}, \mathrm{CH}_{3} \mathrm{CN}$ or DMSO with a JASCO V-750 UV-Visible-NIR spectrophotometer in $10 \mathrm{~mm}$ quartz cells (Hellma); molar extinction coefficients $\varepsilon\left(\mathrm{L} . \mathrm{mol}^{-1} \cdot \mathrm{cm}^{-1}\right)$ are expressed as $\log \varepsilon$. Abbreviation used: sh, shoulder. All spectra can be found in the Supporting Information. Experimental procedures used for DFT calculations are described in the Supporting Information.

\section{General procedure for the preparation of porphyrin cages $\left[\mathrm{Ag}_{4}(1 \mathrm{a}-\mathrm{d})_{2}\right]\left(\mathrm{PF}_{6}\right)_{4}$.}

Porphyrin 4a-d (around 0.04-0.06 mmol) was dissolved in dry $\mathrm{CH}_{3} \mathrm{CN}(10 \mathrm{~mL})$ and degassed with argon for 10 minutes. Then, $\mathrm{Ag}_{2} \mathrm{O}$ (4.0 or $6.0 \mathrm{eq}$ ) was added and the reaction mixture was vigorously stirred under argon at $70^{\circ} \mathrm{C}$ for 24 or 48 hours protected from light. The reaction was monitored by $\mathrm{UV}$-visible absorption spectroscopy (in $\mathrm{CH}_{2} \mathrm{Cl}_{2}$ ) to verify the formation of the porphyrin cage (hypsochromic shift of the Soret absorption band). After cooling at room temperature, the reaction mixture was evaporated. Dichloromethane was added $(10-15 \mathrm{~mL})$ and 
the solution was filtered through a pad of celite. The obtained solution was concentrated under reduced pressure and precipitated from $\mathrm{CH}_{2} \mathrm{Cl}_{2} / \mathrm{Et}_{2} \mathrm{O}$. The solid was filtered off, washed with $\mathrm{Et}_{2} \mathrm{O}$ and dried under vacuum to afford the porphyrin cage $\left[\mathbf{A g}_{4}(\mathbf{1} \mathbf{a - d})_{2}\right]\left(\mathbf{P F}_{6}\right)_{4}$ as a dark solid.

Porphyrin cage $\left[\mathrm{Ag}_{4}\left(\mathbf{1 a}_{2}\right]\left(\mathbf{P F}_{6}\right)_{4}\right.$. The title compound was prepared according to the above general procedure from porphyrin $4 \mathbf{a}(80 \mathrm{mg}, 0.0429 \mathrm{mmol}, 1.0 \mathrm{eq}), \mathrm{Ag}_{2} \mathrm{O}(40 \mathrm{mg}, 0.1718$ mmol, 4.0 eq) and $\mathrm{CH}_{3} \mathrm{CN}(10 \mathrm{~mL})$. The reaction mixture was heated at $75{ }^{\circ} \mathrm{C}$ for 24 hours. The porphyrin cage $\left[\mathbf{A g}_{\mathbf{4}}(\mathbf{1 a})_{2}\right]\left(\mathbf{P F}_{6}\right)_{4}$ was obtained in 77\% yield (59 mg, $\left.0.0165 \mathrm{mmol}\right) .{ }^{1} \mathrm{H}$ NMR (400 MHz, DMSO- $\left.d_{6}\right): \delta=8.41$ (br d, $8 \mathrm{H},{ }^{3} J_{\mathrm{H}, \mathrm{H}}=8.0 \mathrm{~Hz}, \mathrm{H}_{o}$ out $), 8.36$ (br s, $\left.16 \mathrm{H}, \mathrm{H}_{\beta-p y r r}\right), 8.24(\mathrm{~d}, 8 \mathrm{H}$, $\left.{ }^{3} J_{\mathrm{H}, \mathrm{H}}=8.0 \mathrm{~Hz}, \mathrm{H}_{m \text { out }}\right), 8.15\left(\mathrm{~d}, 8 \mathrm{H},{ }^{3} J_{\mathrm{H}, \mathrm{H}}=1.7 \mathrm{~Hz}, \mathrm{H}^{5}\right), 8.00\left(\mathrm{~d}, 8 \mathrm{H},{ }^{3} J_{\mathrm{H}, \mathrm{H}}=1.7 \mathrm{~Hz}, \mathrm{H}^{4}\right), 7.80(\mathrm{br}$ $\left.\mathrm{d}, 8 \mathrm{H},{ }^{3} J_{\mathrm{H}, \mathrm{H}}=8.0 \mathrm{~Hz}, \mathrm{H}_{\text {m in }}\right), 7.66\left(\mathrm{br} \mathrm{d}, 8 \mathrm{H},{ }^{3} J_{\mathrm{H}, \mathrm{H}}=8.0 \mathrm{~Hz}, \mathrm{H}_{o}\right.$ in $), 4.54\left(\mathrm{t}, 16 \mathrm{H},{ }^{3} J_{\mathrm{H}, \mathrm{H}}=7.2 \mathrm{~Hz}, \mathrm{H}_{a}\right)$, 2.15-2.06 (m, 16H, $\left.\mathrm{H}_{b}\right), 1.54-1.36\left(\mathrm{~m}, 24 \mathrm{H}, \mathrm{H}_{c}, \mathrm{H}_{d}, \mathrm{H}_{e}\right) 0.97 \mathrm{ppm}\left(\mathrm{t}, 24 \mathrm{H},{ }^{3} J_{\mathrm{H}, \mathrm{H}}=7.0 \mathrm{~Hz},-\mathrm{CH}_{3}\right)$. ${ }^{13} \mathrm{C}\left\{{ }^{1} \mathrm{H}\right\} \operatorname{NMR}\left(150.9 \mathrm{MHz}, \mathrm{CD}_{2} \mathrm{Cl}_{2}\right): \delta=179.2\left(\mathrm{dd},{ }^{1} J_{107 \mathrm{Ag}, \mathrm{C}}=185 \mathrm{~Hz},{ }^{1} J_{109 \mathrm{Ag}, \mathrm{C}}=216 \mathrm{~Hz}, C_{N H C}\right)$, $148.9\left(C_{\alpha-\text { pyrr }}\right), 143.0\left(C_{p}\right), 140.0\left(C_{i}\right), 136.8\left(C_{o \text { out }}\right), 136.6\left(C_{m \text { out }}\right), 131.7\left(C_{\beta-\text { pyrr }}\right), 123.4\left(C_{\text {o in }}\right)$, $123.1\left(C_{m \text { in }}\right), 122.5\left(C^{4}\right), 122.1\left(C^{5}\right), 118.2\left(C_{\text {meso }}\right), 54.0\left(\mathrm{C}_{a}\right) 32.5\left(\mathrm{C}_{b}\right), 32.2\left(\mathrm{C}_{c}\right), 27.2\left(\mathrm{C}_{d}\right), 23.2$ $\left(\mathrm{C}_{e}\right), 14.4 \mathrm{ppm}\left(-\mathrm{CH}_{3}\right) ;{ }^{19} \mathrm{~F}\left\{{ }^{1} \mathrm{H}\right\} \mathrm{NMR}\left(376.5 \mathrm{MHz}, \mathrm{CD}_{2} \mathrm{Cl}_{2}\right): \delta=-73.8 \mathrm{ppm}\left(\mathrm{d},{ }^{1} J_{\mathrm{F}, \mathrm{P}}=711.5 \mathrm{~Hz}\right)$; ${ }^{31} \mathrm{P}\left\{{ }^{1} \mathrm{H}\right\}$ NMR $\left(162 \mathrm{MHz}, \mathrm{CD}_{2} \mathrm{Cl}_{2}\right): \delta=-144.5 \mathrm{ppm}\left(\mathrm{sept},{ }^{1} \mathrm{~J}_{\mathrm{P}, \mathrm{F}}=711.5 \mathrm{~Hz}\right) . \mathrm{UV}-\mathrm{V}$ is (DMSO): $\lambda_{\max }(\log \varepsilon)=413(5.75), \sim 420-430(\mathrm{sh}), 561$ (4.46), $603 \mathrm{~nm}(4.17)$; HRMS (ESI+): $m / z$ calcd for $\mathrm{C}_{160} \mathrm{H}_{168} \mathrm{~N}_{24} \mathrm{Zn}_{2} \mathrm{Ag}_{4}{ }^{4+}\left[\mathrm{M}-4 \mathrm{PF}_{6}\right]^{4+}:$ 747.2170; found: 747.2181; MS (ESI-): $m / z$ calcd for $\mathrm{PF}_{6}{ }^{-}$: 144.96, found: 144.96 .

Porphyrin cage $\left[\mathrm{Ag}_{4}\left(\mathbf{1 b}_{2}\right]\left(\mathbf{P F}_{6}\right)_{4}\right.$. The title compound was prepared according to the above general procedure from porphyrin $4 \mathbf{b}(80 \mathrm{mg}, 0.0457 \mathrm{mmol}, 1.0 \mathrm{eq}), \mathrm{Ag}_{2} \mathrm{O}$ (43 mg, 0.1828 mmol, $4.0 \mathrm{eq})$ and $\mathrm{CH}_{3} \mathrm{CN}(10 \mathrm{~mL})$. ). The reaction mixture was heated at $75^{\circ} \mathrm{C}$ for 24 hours. The porphyrin cage $\left[\mathbf{A g}_{4}(\mathbf{1 b})_{2}\right]\left(\mathbf{P F}_{6}\right) 4$ was obtained in 68\% yield $(52 \mathrm{mg}, 0.0155 \mathrm{mmol}) .{ }^{1} \mathrm{H}$ NMR $(600$ 
MHz, DMSO-d $\left.)_{6}\right): \delta=8.42\left(\right.$ br d, $\left.8 \mathrm{H},{ }^{3} J_{\mathrm{H}, \mathrm{H}}=7.7 \mathrm{~Hz}, \mathrm{H}_{\text {o out }}\right), 8.36\left(\right.$ br s, $\left.16 \mathrm{H}, \mathrm{H}_{\beta-p y r r}\right), 8.25(\mathrm{dd}, 8 \mathrm{H}$, ${ }^{3} J_{\mathrm{H}, \mathrm{H}}=7.7 \mathrm{~Hz}$ and ${ }^{4} J_{\mathrm{H}, \mathrm{H}}=2.5 \mathrm{~Hz}, \mathrm{H}_{m}$ out $), 8.14\left(\mathrm{~d}, 8 \mathrm{H},{ }^{3} J_{\mathrm{H}, \mathrm{H}}=1.7 \mathrm{~Hz}, \mathrm{H}^{5}\right), 7.98\left(\mathrm{~d}, 8 \mathrm{H},{ }^{3} J_{\mathrm{H}, \mathrm{H}}=1.7\right.$ $\left.\mathrm{Hz}, \mathrm{H}^{4}\right), 7.79\left(\mathrm{br} \mathrm{d}, 8 \mathrm{H},{ }^{3} J_{\mathrm{H}, \mathrm{H}}=7.9 \mathrm{~Hz}\right.$ and ${ }^{4} J_{\mathrm{H}, \mathrm{H}}=2.6 \mathrm{~Hz}, \mathrm{H}_{m}$ in $), 7.66\left(\mathrm{br} \mathrm{d}, 8 \mathrm{H},{ }^{3} J_{\mathrm{H}, \mathrm{H}}=7.9 \mathrm{~Hz}\right.$ and $\left.{ }^{4} J_{\mathrm{H}, \mathrm{H}}=2.6 \mathrm{~Hz}, \mathrm{H}_{\text {o in }}\right), 4.55\left(\mathrm{t}, 16 \mathrm{H},{ }^{3} J_{\mathrm{H}, \mathrm{H}}=7.3 \mathrm{~Hz}, \mathrm{H}_{a}\right), 2.10$ (quint, $\left.16 \mathrm{H},{ }^{3} J_{\mathrm{H}, \mathrm{H}}=7.5 \mathrm{~Hz}, \mathrm{H}_{b}\right), 1.53$ $\left(\right.$ sext, $\left.16 \mathrm{H},{ }^{3} J_{\mathrm{H}, \mathrm{H}}=7.5 \mathrm{~Hz}, \mathrm{H}_{c}\right), 1.09 \mathrm{ppm}\left(\mathrm{t}, 24 \mathrm{H},{ }^{3} J_{\mathrm{H}, \mathrm{H}}=7.5 \mathrm{~Hz},-\mathrm{C} H_{3}\right) .{ }^{13} \mathrm{C}\left\{{ }^{1} \mathrm{H}\right\} \mathrm{NMR}(150.9$ MHz, DMSO- $\left.d_{6}\right): \delta=173.4,\left(C_{N H C}\right), 148.9\left(C_{\alpha-p y r r}\right), 142.7\left(C_{p}\right), 138.9\left(C_{i}\right), 135.2\left(C_{o \text { out }}\right), 134.8$ $\left(C_{\text {o in }}\right), 130.2\left(C_{\beta-p y r r}\right), 123.4\left(C^{4}\right), 122.9\left(C^{5}\right), 121.5\left(C_{m \text { in }}\right), 121.0\left(C_{m \text { out }}\right), 116.5\left(C_{\text {meso }}\right), 51.9\left(\mathrm{C}_{a}\right)$ $33.5\left(\mathrm{C}_{b}\right), 19.6\left(\mathrm{C}_{c}\right), 13.8 \mathrm{ppm}\left(-\mathrm{CH}_{3}\right), \mathrm{C}_{\mathrm{NHC}}$ not observed. ${ }^{19} \mathrm{~F}\left\{{ }^{1} \mathrm{H}\right\}$ NMR (376.5 MHz, DMSO$\left.d_{6}\right): \delta=-70.1 \mathrm{ppm}\left(\mathrm{d},{ }^{1} J_{\mathrm{F}, \mathrm{P}}=711.7 \mathrm{~Hz}\right) ;{ }^{31} \mathrm{P}\left\{{ }^{1} \mathrm{H}\right\}$ NMR $\left(162 \mathrm{MHz}, \mathrm{DMSO}-d_{6}\right): \delta=-144.0 \mathrm{ppm}$ (sept, ${ }^{1} J_{\mathrm{P}, \mathrm{F}}=711.4 \mathrm{~Hz}$ ); UV-Vis (DMSO): $\lambda_{\max }(\log \varepsilon)=413$ (5.51), 420-430 (sh), 562 (4.51), $603 \mathrm{~nm}$ (4.35); HRMS (ESI+): $m / z$ calcd for $\mathrm{C}_{144} \mathrm{H}_{136} \mathrm{~N}_{24} \mathrm{Zn}_{2} \mathrm{Ag}_{4}{ }^{4+}\left[\mathrm{M}-4 \mathrm{PF}_{6}\right]^{4+}:$ 691.1534; found: 691.1505; MS (ESI-): $m / z$ calcd for $\mathrm{PF}_{6}^{-}: 144.96$, found: 144.96.

Porphyrin cage $\left[\mathrm{Ag}_{4}\left(\mathbf{1 c}_{2}\right)_{(\mathbf{P F}}\right)_{4}$. The title compound was prepared according to the above general procedure from porphyrin $4 \mathrm{c}(100 \mathrm{mg}, 0.0521 \mathrm{mmol}, 1.0 \mathrm{eq}) . \mathrm{Ag}_{2} \mathrm{O}(75 \mathrm{mg}, 0.3126$ mmol, 6.0 eq) and $\mathrm{CH}_{3} \mathrm{CN}(10 \mathrm{~mL})$. The reaction mixture was heated at $75^{\circ} \mathrm{C}$ for 48 hours. Indeed, extended reaction time (48 hours) and more $\mathrm{Ag}_{2} \mathrm{O}$ (6 eq) were needed to successfully achieve the synthesis of porphyrin cage $\left[\mathbf{A g}_{\mathbf{4}}(\mathbf{1 c})_{2}\right]\left(\mathbf{P F}_{6}\right)_{4}$ in $71 \%$ yield $(68 \mathrm{mg}, 0.0185 \mathrm{mmol}) .{ }^{1} \mathrm{H}$ NMR $(400$ $\left.\mathrm{MHz}, \mathrm{CD}_{2} \mathrm{Cl}_{2}\right): \delta=7.88\left(\mathrm{~s}, 16 \mathrm{H}, \mathrm{H}_{\beta-p y r r}\right), 7.64\left(\mathrm{~d} \mathrm{br}, 8 \mathrm{H},{ }^{3} J_{\mathrm{H}, \mathrm{H}}=7.5 \mathrm{~Hz}, \mathrm{H}_{m}\right.$ in $), 7.52\left(\mathrm{~d} \mathrm{br}, 8 \mathrm{H},{ }^{3} J_{\mathrm{H}, \mathrm{H}}\right.$ $\left.=7.5 \mathrm{~Hz}, \mathrm{H}_{\text {o out }}\right), 7.41\left(\mathrm{~d} \mathrm{br}, 8 \mathrm{H},{ }^{3} J_{\mathrm{H}, \mathrm{H}}=7.5 \mathrm{~Hz} \mathrm{H}_{m}\right.$ out $), 7.34\left(\mathrm{~s}\right.$ br, $\left.\mathrm{H}^{4}\right), 7.33\left(\mathrm{~s} \mathrm{br}, \mathrm{H}^{5}\right), 7.22(\mathrm{~d}$ br, $8 \mathrm{H},{ }^{3} J_{\mathrm{H}, \mathrm{H}}=7.5 \mathrm{~Hz}, \mathrm{H}_{o}$ in $), 5.74\left(\mathrm{~s}, 16 \mathrm{H}, \mathrm{CH}_{2}\right), 4.41\left(\mathrm{t} \mathrm{br}, 16 \mathrm{H},{ }^{3} J_{\mathrm{H}, \mathrm{H}}=7.0 \mathrm{~Hz}, \mathrm{H}_{a}\right.$ ), 2.09 (quint br, $\left.16 \mathrm{H},{ }^{3} J_{\mathrm{H}, \mathrm{H}}=7.0 \mathrm{~Hz}, \mathrm{H}_{b}\right), 1.60-1.40\left(\mathrm{~m}, 48 \mathrm{H}, \mathrm{H}_{c}, \mathrm{H}_{d}, \mathrm{H}_{e}\right), 0.97 \mathrm{ppm}\left(\mathrm{t}, 24 \mathrm{H},{ }^{3} J_{\mathrm{H}, \mathrm{H}}=7.0 \mathrm{~Hz},-\mathrm{CH}_{3}\right)$. ${ }^{13} \mathrm{C}\left\{{ }^{1} \mathrm{H}\right\} \mathrm{NMR}\left(125.8 \mathrm{MHz}, \mathrm{CD}_{2} \mathrm{Cl}_{2}\right): 180.8 \mathrm{ppm}\left(\mathrm{dd},{ }^{1} J_{107 \mathrm{Ag}, \mathrm{C}}=185 \mathrm{~Hz},{ }^{1} J_{109 \mathrm{Ag}, \mathrm{C}}=216 \mathrm{~Hz}, C_{N H C}\right)$, $148.6\left(C_{\alpha-p y r r}\right), 142.2\left(\mathrm{C}_{p}\right), 136.5\left(\mathrm{C}_{i}\right), 135.9\left(\mathrm{C}_{\text {o in }}\right), 135.6\left(\mathrm{C}_{\text {o out }}\right), 130.9\left(C_{\beta-p y r r}\right), 126.2\left(\mathrm{C}_{m}\right.$ out $)$, 
$125.9\left(C_{\text {m in }}\right), 122.9\left(\mathrm{C}_{4}\right), 122.7\left(\mathrm{C}_{5}\right), 119.6\left(\mathrm{C}_{\text {meso }}\right), 56.2\left(\mathrm{CH}_{2}\right), 53.1\left(\mathrm{C}_{a}\right), 32.3\left(\mathrm{C}_{b}\right), 32.0\left(\mathrm{C}_{c}\right), 27.0$ $\left(\mathrm{C}_{d}\right), 23.2\left(\mathrm{C}_{e}\right), 14.4 \mathrm{ppm}\left(\mathrm{CH}_{3}\right) ;{ }^{19} \mathrm{~F}\left\{{ }^{1} \mathrm{H}\right\} \mathrm{NMR}\left(376.5 \mathrm{MHz}, \mathrm{CD}_{3} \mathrm{CN}\right): \delta=-72.8 \mathrm{ppm}\left(\mathrm{d},{ }^{1} J_{\mathrm{F}, \mathrm{P}}=\right.$ $706.7 \mathrm{~Hz}) ;{ }^{31} \mathrm{P}\left\{{ }^{1} \mathrm{H}\right\} \mathrm{NMR}\left(162 \mathrm{MHz}, \mathrm{CD}_{3} \mathrm{CN}\right): \delta=-144.4 \mathrm{ppm}\left(\mathrm{sept},{ }^{1} J_{\mathrm{P}, \mathrm{F}}=706.7 \mathrm{~Hz}\right) ; \mathrm{UV}-\mathrm{Vis}$ (DMSO): $\lambda_{\max }(\log \varepsilon)=426$ (5.82), $561(4.46), 602 \mathrm{~nm}(4.31)$; HRMS (ESI+): $m / z$ calcd for $\mathrm{C}_{168} \mathrm{H}_{184} \mathrm{~N}_{24} \mathrm{Zn}_{2} \mathrm{Ag}_{4}{ }^{4+}\left[\mathrm{M}-4 \mathrm{PF}_{6}\right]^{4+}:$ 775.2463; found: 775.2479; MS (ESI-): $m / z$ calcd for $\mathrm{PF}_{6}{ }^{-}$: 144.96, found: 144.96 .

Porphyrin cage $\left[\mathrm{Ag}_{4}(\mathbf{1 d})_{2}\right]\left(\mathbf{P F}_{6}\right)_{4}$. The title compound was prepared according to the above general procedure from porphyrin $\mathbf{4 d}$ (80 mg, $0.0443 \mathrm{mmol}, 1.0 \mathrm{eq}), \mathrm{Ag}_{2} \mathrm{O}$ (41 mg, 0.1771 mmol, 4 eq) and $\mathrm{CH}_{3} \mathrm{CN}(10 \mathrm{~mL})$. The porphyrin cage $\left[\mathbf{A g}_{4}(\mathbf{1} \mathbf{d})_{2}\right]\left(\mathbf{P F}_{6}\right)_{4}$ was obtained in $82 \%$ yield (63 mg, $0.0182 \mathrm{mmol}) .{ }^{1} \mathrm{H}$ NMR (600 MHz, $\mathrm{CD}_{3} \mathrm{CN}$ ): $\delta=8.24$ (br s, $16 \mathrm{H}, \mathrm{H}_{\beta-p y r r}$ ), 8.07 (br d, $8 \mathrm{H}$, $\left.{ }^{3} J_{\mathrm{H}, \mathrm{H}}=7.3 \mathrm{~Hz}, \mathrm{H}_{\text {o out }}\right), 7.82\left(\mathrm{br} \mathrm{d}, 8 \mathrm{H},{ }^{3} J_{\mathrm{H}, \mathrm{H}}=7.3 \mathrm{~Hz}, \mathrm{H}_{m}\right.$ out $), 7.77\left(\mathrm{~d}, 8 \mathrm{H},{ }^{3} J_{\mathrm{H}, \mathrm{H}}=7.3 \mathrm{~Hz}, \mathrm{H}_{m}\right.$ in $)$, $7.53\left(\mathrm{~d}, 8 \mathrm{H},{ }^{3} J_{\mathrm{H}, \mathrm{H}}=1.9 \mathrm{~Hz}, \mathrm{H}^{5}\right), 7.42\left(\mathrm{~d}, 8 \mathrm{H},{ }^{3} J_{\mathrm{H}, \mathrm{H}}=1.9 \mathrm{~Hz}, \mathrm{H}^{4}\right), 7.41\left(\mathrm{br} \mathrm{d}, 8 \mathrm{H},{ }^{3} J_{\mathrm{H}, \mathrm{H}}=7.3 \mathrm{~Hz}, \mathrm{H}_{o}\right.$ in), 5.69 (s, 16H, $\mathrm{CH}_{2}$ ), $4.40\left(\mathrm{t}, 16 \mathrm{H},{ }^{3} J_{\mathrm{H}, \mathrm{H}}=7.1 \mathrm{~Hz}, \mathrm{H}_{a}\right.$ ), 2.00 (quint, $16 \mathrm{H},{ }^{3} J_{\mathrm{H}, \mathrm{H}}=7.1 \mathrm{~Hz}, \mathrm{H}_{b}$ ), 1.49 (sext, $\left.16 \mathrm{H},{ }^{3} J_{\mathrm{H}, \mathrm{H}}=7.1 \mathrm{~Hz}, \mathrm{H}_{c}\right), 1.01 \mathrm{ppm}\left(\mathrm{t}, 24 \mathrm{H},{ }^{3} \mathrm{~J}_{\mathrm{H}, \mathrm{H}}=7.1 \mathrm{~Hz},-\mathrm{CH}_{3}\right) ;{ }^{13} \mathrm{C}\left\{{ }^{1} \mathrm{H}\right\} \mathrm{NMR}(150.9$ $\left.\mathrm{MHz}, \mathrm{CD}_{3} \mathrm{CN}\right): 149.9\left(C_{\alpha-p y r r}\right), 144.1\left(\mathrm{C}_{p}\right), 136.7\left(\mathrm{C}_{i}\right), 136.0\left(\mathrm{C}_{\text {o in }}\right), 134.4\left(\mathrm{C}_{\text {o out }}\right), 131.9\left(C_{\beta-p y r r}\right)$, 127.6 $\left(\mathrm{C}_{\text {m out }}\right), 126.3\left(C_{m \text { in }}\right), 123.7\left(\mathrm{C}_{4}\right), 122.6\left(\mathrm{C}_{5}\right), 119.9\left(\mathrm{C}_{m e s o}\right), 56.2\left(\mathrm{CH}_{2}\right), 52.5\left(\mathrm{C}_{a}\right), 34.4\left(\mathrm{C}_{b}\right)$, $20.6\left(\mathrm{C}_{c}\right), 14.0 \mathrm{ppm}\left(\mathrm{CH}_{3}\right), \mathrm{C}_{\mathrm{NHC}}$ not observed; ${ }^{19} \mathrm{~F}\left\{{ }^{1} \mathrm{H}\right\} \mathrm{NMR}\left(376.5 \mathrm{MHz}, \mathrm{CD}_{3} \mathrm{CN}\right): \delta=-73.9$ $\operatorname{ppm}\left(\mathrm{d},{ }^{1} J_{\mathrm{F}, \mathrm{P}}=706.5 \mathrm{~Hz}\right) ;{ }^{31} \mathrm{P}\left\{{ }^{1} \mathrm{H}\right\} \mathrm{NMR}\left(162 \mathrm{MHz}, \mathrm{CD}_{3} \mathrm{CN}\right): \delta=-144.7 \mathrm{ppm}\left(\mathrm{sept},{ }^{1} J_{\mathrm{P}, \mathrm{F}}=706.5\right.$ Hz); UV-Vis (DMSO): $\lambda_{\max }(\log \varepsilon)=422$ (5.83), 564 (4.69), $604 \mathrm{~nm}$ (4.52); HRMS (ESI+): $m / z$ calcd for $\mathrm{C}_{152} \mathrm{H}_{152} \mathrm{~N}_{24} \mathrm{Zn}_{2} \mathrm{Ag}_{4}{ }^{4+}\left[\mathrm{M}-4 \mathrm{PF}_{6}\right]^{4+}$ : 719.1857; found: 719.1875; MS (ESI-): $m / z$ calcd for $\mathrm{PF}_{6}{ }^{-}: 144.96$; found: 144.96 . 


\section{General procedure for the preparation of porphyrin cages $\left[\mathrm{Au}_{4}\left(\mathbf{1 a}_{-\mathrm{d}}\right)_{2}\right]\left(\mathrm{PF}_{6}\right)_{4}$.}

Porphyrin cages $\left[\mathbf{A g}_{4}(\mathbf{1} \mathbf{a}-\mathbf{d})_{2}\right]\left(\mathbf{P F}_{6}\right)_{4}$ (around 0.01-0.02 mmol) was dissolved in dry $\mathrm{CH}_{3} \mathrm{CN}(5 \mathrm{~mL}$ ) and degassed with argon for 10 minutes. Then, [AuCl(tht)] (6 eq) was added and the reaction mixture was vigorously stirred under argon and protected from light at room temperature for 24 hours. The reaction was monitored by $\operatorname{ESI}-\mathrm{TOF}(+)$ mass spectrometry. Once finished, the reaction mixture was evaporated. Dichloromethane was added (10-15 mL) and the solution was filtered through a pad of celite. The obtained solution was concentrated under reduced pressure and precipitated from $\mathrm{CH}_{2} \mathrm{Cl}_{2} / \mathrm{Et}_{2} \mathrm{O}$. The solid was filtered off, washed with $\mathrm{Et}_{2} \mathrm{O}$ and dried under vacuum to afford the porphyrin cage $\left[\mathbf{A g}_{4}(\mathbf{1} \mathbf{a}-\mathbf{d})_{2}\right]\left(\mathbf{P F}_{6}\right)_{4}$ as a dark solid.

Porphyrin cage $\left[\mathbf{A u}_{4}\left(\mathbf{1}_{2}\right)_{2}\right]\left(\mathbf{P F}_{6}\right)_{4}$. The title compound was prepared according to the above general procedure from the porphyrin cage $\left[\mathbf{A g}_{4}\left(\mathbf{1}_{\mathbf{a}}\right)_{2}\right]\left(\mathbf{P F}_{6}\right)_{4}(42 \mathrm{mg}, 0.0117 \mathrm{mmol}, 1.0 \mathrm{eq})$, [AuCl(tht)] (23 mg, $0.0702 \mathrm{mmol}, 6.0 \mathrm{eq})$, and $\mathrm{CH}_{3} \mathrm{CN}(5 \mathrm{~mL})$. The porphyrin cage [Au4(1) $\left.\mathbf{1 a}_{2}\right]\left(\mathbf{P F}_{6}\right)_{4}$ was obtained in 68\% yield (32 $\left.\mathrm{mg}, 0.0081 \mathrm{mmol}\right) .{ }^{1} \mathrm{H}$ NMR (400 MHz, DMSO$\left.d_{6}\right): \delta=8.39$ (br s, $\left.16 \mathrm{H}, \mathrm{H}_{\beta-p y r r}\right), 8.37\left(\mathrm{dd}, 8 \mathrm{H},{ }^{3} J_{\mathrm{H}, \mathrm{H}}=7.8 \mathrm{~Hz}\right.$ and $\left.{ }^{4} J_{\mathrm{H}, \mathrm{H}}=2.5 \mathrm{~Hz}, \mathrm{H}_{\text {o out }}\right), 8.20(\mathrm{dd}$ $, 8 \mathrm{H},{ }^{3} J_{\mathrm{H}, \mathrm{H}}=7.8 \mathrm{~Hz}$ and ${ }^{4} J_{\mathrm{H}, \mathrm{H}}=2.5 \mathrm{~Hz}, \mathrm{H}_{m}$ out $), 8.16\left(\mathrm{~d}, 8 \mathrm{H},{ }^{3} J_{\mathrm{H}, \mathrm{H}}=1.7 \mathrm{~Hz}, \mathrm{H}^{5}\right), 8.06\left(\mathrm{~d}, 8 \mathrm{H},{ }^{3} J_{\mathrm{H}, \mathrm{H}}\right.$ $\left.=1.7 \mathrm{~Hz}, \mathrm{H}^{4}\right), 7.83\left(\mathrm{dd}, 8 \mathrm{H},{ }^{3} J_{\mathrm{H}, \mathrm{H}}=7.8 \mathrm{~Hz}\right.$ and ${ }^{4} J_{\mathrm{H}, \mathrm{H}}=2.5 \mathrm{~Hz}, \mathrm{H}_{m}$ in $), 7.74\left(\mathrm{dd}, 8 \mathrm{H},{ }^{3} J_{\mathrm{H}, \mathrm{H}}=7.8 \mathrm{~Hz}\right.$ and ${ }^{4} J_{\mathrm{H}, \mathrm{H}}=2.5 \mathrm{~Hz}, \mathrm{H}_{o}$ in $), 4.63\left(\mathrm{t}, 16 \mathrm{H},{ }^{3} J_{\mathrm{H}, \mathrm{H}}=7.5 \mathrm{~Hz}, \mathrm{H}_{a}\right), 2.14\left(\mathrm{~m}, 16 \mathrm{H}, \mathrm{H}_{b}\right), 1.51-1.41(\mathrm{~m}, 48 \mathrm{H}$, $\left.\mathrm{H}_{c}, \mathrm{H}_{d}, \mathrm{H}_{e}\right), 0.98 \mathrm{ppm}\left(\mathrm{t}, 24 \mathrm{H},{ }^{3} \mathrm{~J}_{\mathrm{H}, \mathrm{H}}=7.0 \mathrm{~Hz},-\mathrm{CH}_{3}\right) ;{ }^{13} \mathrm{C}\left\{{ }^{1} \mathrm{H}\right\}$ NMR $\left(150.9 \mathrm{MHz}, \mathrm{DMSO}-d_{6}\right): \delta=$ $180.7\left(C_{N H C}\right), 147.2\left(C_{\alpha-p y r r}\right), 142.9\left(C_{p}\right), 138.0\left(C_{i}\right), 135.6\left(\mathrm{C}_{\text {o out }}\right), 134.9\left(\mathrm{C}_{\text {o in }}\right), 130.3\left(C_{\beta-p y r r}\right)$, $123.8\left(\mathrm{C}_{m \text { in }}\right), 123.7\left(\mathrm{C}_{m \text { out }}\right), 122.3\left(C^{4}\right), 122.1\left(C^{5}\right), 116.6\left(C_{\text {meso }}\right), 55.0\left(\mathrm{C}_{a}\right) 31.4\left(\mathrm{C}_{b}\right), 31.2\left(\mathrm{C}_{c}\right)$, $26.0\left(\mathrm{C}_{d}\right), 22.2\left(\mathrm{C}_{e}\right), 14.1 \mathrm{ppm}\left(-\mathrm{CH}_{3}\right) ;{ }^{19} \mathrm{~F}\left\{{ }^{1} \mathrm{H}\right\}$ NMR (376.5 MHz, DMSO- $\left.d_{6}\right): \delta=-71.1 \mathrm{ppm}(\mathrm{d}$, $\left.{ }^{1} J_{\mathrm{F}, \mathrm{P}}=711.5 \mathrm{~Hz}\right) ;{ }^{31} \mathrm{P}\left\{{ }^{1} \mathrm{H}\right\}$ NMR $\left(162 \mathrm{MHz}, \mathrm{DMSO}-d_{6}\right): \delta=-144.3 \mathrm{ppm}\left(\mathrm{sept},{ }^{1} J_{\mathrm{P}, \mathrm{F}}=711.5 \mathrm{~Hz}\right) ;$ UV-Vis (DMSO): $\lambda_{\max }(\log \varepsilon)=414$ (5.53), 562 (4.43), $604 \mathrm{~nm}$ (4.19); HRMS (ESI+): $m / z$ calcd 
for $\mathrm{C}_{160} \mathrm{H}_{168} \mathrm{~N}_{24} \mathrm{Zn}_{2} \mathrm{Au}_{4}{ }^{4+}\left[\mathrm{M}-4 \mathrm{PF}_{6}\right]^{4+}$ : 836.2786; found: 836.2804 ; MS (ESI-): $m / z$ calcd for $\mathrm{PF}_{6}{ }^{-}$: 144.96, found: 144.96 .

Porphyrin cage $\left[\mathrm{Au}_{4}\left(\mathbf{1 b}_{2}\right)_{\mathbf{2}} \mathbf{P F}_{6}\right)_{4}$. The title compound was prepared according to the above general procedure from the porphyrin cage $\left[\mathbf{A g}_{\mathbf{4}}(\mathbf{1 b})_{2}\right]\left(\mathbf{P F}_{\mathbf{6}}\right)_{\mathbf{4}}(60 \mathrm{mg}, 0.0179 \mathrm{mmol}, 1.0 \mathrm{eq})$, [AuCl(tht)] (35 mg, $0.1074 \mathrm{mmol}, 6.0 \mathrm{eq}$ ), and $\mathrm{CH}_{3} \mathrm{CN}(5 \mathrm{~mL})$. The porphyrin cage [Au4(1c)2](PF6)4 was obtained in 60\% yield (40 mg, $0.0107 \mathrm{mmol}) .{ }^{1} \mathrm{H}$ NMR (600 MHz, DMSO$\left.d_{6}\right): \delta=8.39$ (br s, $\left.16 \mathrm{H}, \mathrm{H}_{\beta-\text { pyrr }}\right), 8.38\left(\mathrm{dd}, 8 \mathrm{H},{ }^{3} J_{\mathrm{H}, \mathrm{H}}=7.8 \mathrm{~Hz}\right.$ and ${ }^{4} J_{\mathrm{H}, \mathrm{H}}=2.4 \mathrm{~Hz}, \mathrm{H}_{o}$ out $), 8.20(\mathrm{dd}$ $, 8 \mathrm{H},{ }^{3} J_{\mathrm{H}, \mathrm{H}}=7.8 \mathrm{~Hz}$ and ${ }^{4} J_{\mathrm{H}, \mathrm{H}}=2.4 \mathrm{~Hz}, \mathrm{H}_{m}$ out $), 8.15\left(\mathrm{~d}, 8 \mathrm{H},{ }^{3} J_{\mathrm{H}, \mathrm{H}}=1.7 \mathrm{~Hz}, \mathrm{H}^{5}\right), 8.04\left(\mathrm{~d}, 8 \mathrm{H},{ }^{3} J_{\mathrm{H}, \mathrm{H}}\right.$ $\left.=1.7 \mathrm{~Hz}, \mathrm{H}^{4}\right), 7.82\left(\mathrm{dd}, 8 \mathrm{H},{ }^{3} J_{\mathrm{H}, \mathrm{H}}=7.9 \mathrm{~Hz}\right.$ and $\left.{ }^{4} J_{\mathrm{H}, \mathrm{H}}=2.4 \mathrm{~Hz}, \mathrm{H}_{m \text { in }}\right), 7.72\left(\mathrm{dd}, 8 \mathrm{H},{ }^{3} J_{\mathrm{H}, \mathrm{H}}=7.9 \mathrm{~Hz}\right.$ and ${ }^{4} J_{\mathrm{H}, \mathrm{H}}=2.4 \mathrm{~Hz}, \mathrm{H}_{o}$ in $), 4.63\left(\mathrm{t}, 16 \mathrm{H},{ }^{3} J_{\mathrm{H}, \mathrm{H}}=7.4 \mathrm{~Hz}, \mathrm{H}_{a}\right.$ ), 2.13 (quint, $16 \mathrm{H},{ }^{3} J_{\mathrm{H}, \mathrm{H}}=7.5 \mathrm{~Hz}, \mathrm{H}_{b}$ ), $1.54\left(\mathrm{sext}, 16 \mathrm{H},{ }^{3} J_{\mathrm{H}, \mathrm{H}}=7.4 \mathrm{~Hz}, \mathrm{H}_{c}\right), 1.09 \mathrm{ppm}\left(\mathrm{t}, 24 \mathrm{H},{ }^{3} J_{\mathrm{H}, \mathrm{H}}=7.4 \mathrm{~Hz},-\mathrm{CH}_{3}\right) .{ }^{13} \mathrm{C}\left\{{ }^{1} \mathrm{H}\right\} \mathrm{NMR}(150.9$ MHz, DMSO-d $\left.d_{6}\right): \delta=180.8\left(C_{N H C}\right), 147.2\left(C_{\alpha-p y r r}\right), 142.9\left(C_{p}\right), 137.9\left(C_{i}\right), 135.6\left(\mathrm{C}_{o \text { out }}\right), 134.9\left(\mathrm{C}_{o}\right.$ in $), 130.3\left(C_{\beta-\text { pyrr }}\right), 123.7\left(\mathrm{C}_{\text {m in }}\right), 123.6\left(\mathrm{C}_{\text {m out }}\right), 122.2\left(C^{4}\right), 122.0\left(C^{5}\right), 116.5\left(C_{\text {meso }}\right), 51.2\left(\mathrm{C}_{a}\right) 33.3$ $\left(\mathrm{C}_{b}\right), 19.5\left(\mathrm{C}_{c}\right), 13.8 \mathrm{ppm}\left(-\mathrm{CH}_{3}\right)$; NMR $\left(376.5 \mathrm{MHz}, \mathrm{DMSO}-d_{6}\right): \delta=-70.1 \mathrm{ppm}\left(\mathrm{d},{ }^{1} J_{\mathrm{F}, \mathrm{P}}=711.8\right.$ $\mathrm{Hz}) ;{ }^{31} \mathrm{P}\left\{{ }^{1} \mathrm{H}\right\}$ NMR (162 MHz, DMSO- $\left.d_{6}\right): \delta=-144.0 \mathrm{ppm}\left(\mathrm{sept},{ }^{1} J_{\mathrm{P}, \mathrm{F}}=711.8 \mathrm{~Hz}\right.$ ); UV-Vis (DMSO): $\lambda_{\max }(\log \varepsilon)=414$ (5.65), 562 (4.29), $603 \mathrm{~nm}$ (3.95); HRMS (ESI+): $m / z$ calcd for $\mathrm{C}_{144} \mathrm{H}_{136} \mathrm{~N}_{24} \mathrm{Zn}_{2} \mathrm{Ag}_{4}{ }^{4+}\left[\mathrm{M}-4 \mathrm{PF}_{6}\right]^{4+}:$ 780.2152; found: 780.2101 ; MS (ESI-): $m / z$ calcd for $\mathrm{PF}_{6}{ }^{-}$: 144.96, found: 144.96 .

Porphyrin cage $\left[\mathrm{Au}_{4}\left(\mathbf{1 c}_{2}\right)_{2} \mathbf{P F}_{6}\right)_{4}$. The title compound was prepared according to the above general procedure from the porphyrin cage $\left[\mathbf{A g}_{4}(\mathbf{1 c})_{2}\right]\left(\mathbf{P F}_{6}\right)_{4}(55 \mathrm{mg}, 0.0149 \mathrm{mmol}, 1.0 \mathrm{eq})$, [AuCl(tht)] (29 mg, $0.0894 \mathrm{mmol}, 6.0 \mathrm{eq})$, and $\mathrm{CH}_{3} \mathrm{CN}(5 \mathrm{~mL})$. The porphyrin cage [Au4(1c)2](PF6)4 was obtained in 72\% yield (43 mg, $0.0107 \mathrm{mmol}) .{ }^{1} \mathrm{H}$ NMR $\left(600 \mathrm{MHz}, \mathrm{CD}_{2} \mathrm{Cl}_{2}\right)$ : 
$\delta=7.97\left(\mathrm{~s}, 16 \mathrm{H}, \mathrm{H}_{\beta-p y r r}\right), 7.65\left(\mathrm{dd}, 8 \mathrm{H},{ }^{3} J_{\mathrm{H}, \mathrm{H}}=7.5 \mathrm{~Hz}\right.$ and $\left.{ }^{4} J_{\mathrm{H}, \mathrm{H}}=1.5 \mathrm{~Hz}, \mathrm{H}_{m i n}\right), 7.56\left(\mathrm{dd}, 8 \mathrm{H},{ }^{3} J_{\mathrm{H}, \mathrm{H}}\right.$ $=7.5 \mathrm{~Hz}$ and $\left.{ }^{4} J_{\mathrm{H}, \mathrm{H}}=1.8 \mathrm{~Hz}, \mathrm{H}_{\text {o out }}\right), 7.41\left(\mathrm{dd}, 8 \mathrm{H},{ }^{3} J_{\mathrm{H}, \mathrm{H}}=7.5 \mathrm{~Hz}\right.$ and ${ }^{4} J_{\mathrm{H}, \mathrm{H}}=1.5 \mathrm{~Hz}, \mathrm{H}_{m}$ out $), 7.36$ $\left(\mathrm{d}, 8 \mathrm{H},{ }^{3} J_{\mathrm{H}, \mathrm{H}}=1.7 \mathrm{~Hz}, \mathrm{H}^{4}\right), 7.34\left(\mathrm{~d}, 8 \mathrm{H},{ }^{3} J_{\mathrm{H}, \mathrm{H}}=1.7 \mathrm{~Hz}, \mathrm{H}^{5}\right), 7.22\left(\mathrm{dd}, 8 \mathrm{H},{ }^{3} J_{\mathrm{H}, \mathrm{H}}=7.5 \mathrm{~Hz}\right.$ and ${ }^{4} J_{\mathrm{H}, \mathrm{H}}$ $\left.=1.8 \mathrm{~Hz}, \mathrm{H}_{\text {o in }}\right), 5.81\left(\mathrm{~s}, 16 \mathrm{H}, \mathrm{CH}_{2}\right), 4.49\left(\mathrm{t}, 16 \mathrm{H},{ }^{3} J_{\mathrm{H}, \mathrm{H}}=7.0 \mathrm{~Hz}, \mathrm{H}_{a}\right), 2.13$ (quint, $16 \mathrm{H},{ }^{3} \mathrm{~J}_{\mathrm{H}, \mathrm{H}}=7.0$ $\left.\mathrm{Hz}, \mathrm{H}_{b}\right), 1.60-1.40\left(\mathrm{~m}, 48 \mathrm{H}, \mathrm{H}_{c}, \mathrm{H}_{d}, \mathrm{H}_{e}\right), 0.98 \mathrm{ppm}\left(\mathrm{t}, 24 \mathrm{H},{ }^{3} \mathrm{~J}_{\mathrm{H}, \mathrm{H}}=7.0 \mathrm{~Hz},-\mathrm{CH}_{3}\right) ;{ }^{13} \mathrm{C}\left\{{ }^{1} \mathrm{H}\right\} \mathrm{NMR}$ $\left(150.9 \mathrm{MHz}, \mathrm{CD}_{3} \mathrm{CN}\right): \delta=184.8\left(C_{N H C}\right), 148.7\left(C_{\alpha-p y r r}\right), 142.3\left(\mathrm{C}_{i}\right), 136.9\left(\mathrm{C}_{o \text { in }}\right), 135.6\left(\mathrm{C}_{p}\right), 135.5$ $\left(\mathrm{C}_{\text {o out }}\right), 131.1\left(C_{\beta-p y r r}\right), 126.1\left(\mathrm{C}_{\text {min }}\right), 126.1\left(\mathrm{C}_{\text {mout }}\right), 123.0\left(\mathrm{C}_{4}\right.$ or $\left.\mathrm{C}_{5}\right), 122.9\left(\mathrm{C}_{4}\right.$ or $\left.\mathrm{C}_{5}\right), 119.7\left(\mathrm{C}_{\text {meso }}\right)$, $55.6\left(\mathrm{CH}_{2}\right), 52.5\left(\mathrm{C}_{a}\right), 32.2\left(\mathrm{C}_{b}\right), 32.1\left(\mathrm{C}_{c}\right), 27.0\left(\mathrm{C}_{d}\right), 23.2\left(\mathrm{C}_{e}\right), 14.5 \mathrm{ppm}\left(\mathrm{CH}_{3}\right) ;{ }^{19} \mathrm{~F}\left\{{ }^{1} \mathrm{H}\right\} \mathrm{NMR}$ $\left(376.5 \mathrm{MHz}, \mathrm{CD}_{3} \mathrm{CN}\right): \delta=-72.6 \mathrm{ppm}\left(\mathrm{d},{ }^{1} J_{\mathrm{F}, \mathrm{P}}=706.9 \mathrm{~Hz}\right) ;{ }^{31} \mathrm{P}\left\{{ }^{1} \mathrm{H}\right\} \mathrm{NMR}\left(162 \mathrm{MHz}, \mathrm{CD}_{3} \mathrm{CN}\right): \delta$ $=-144.1 \mathrm{ppm}\left(\mathrm{sept},{ }^{1} J_{\mathrm{P}, \mathrm{F}}=706.9 \mathrm{~Hz}\right)$; UV-Vis $(\mathrm{DMSO}): \lambda_{\max }(\log \varepsilon)=425$ (5.54), 562 (4.39), 602 $\mathrm{nm}$ (4.23); HRMS (ESI+): $m / z$ calcd for $\mathrm{C}_{168} \mathrm{H}_{184} \mathrm{~N}_{24} \mathrm{Zn}_{2} \mathrm{Au}_{4}{ }^{4+}\left[\mathrm{M}-4 \mathrm{PF}_{6}\right]^{4+}:$ 864.5595; found: 864.5514; MS (ESI-): $m / z$ calcd for $\mathrm{PF}_{6}^{-}: 144.96$, found: 144.96 .

Porphyrin cage $\left[\mathbf{A u}_{4}(\mathbf{1 d})_{2}\right]\left(\mathbf{P F}_{6}\right)_{4}$. The title compound was prepared according to the above general procedure from the porphyrin cage $\left[\mathbf{A g}_{4}(\mathbf{1 d})_{2}\right]\left(\mathbf{P F}_{6}\right) 4(36 \mathrm{mg}, 0.0104 \mathrm{mmol}, 1.0 \mathrm{eq})$, [AuCl(tht)] $(20 \mathrm{mg}, 0.0624 \mathrm{mmol}, 6.0 \mathrm{eq})$, and $\mathrm{CH}_{3} \mathrm{CN}(5 \mathrm{~mL})$. The porphyrin cage [Au4 $\left.\mathbf{A}(\mathbf{1 d})_{2}\right]\left(\mathbf{P F}_{6}\right)_{4}$ was obtained in $86 \%$ yield $(34 \mathrm{mg}, 0.0089 \mathrm{mmol}) .{ }^{1} \mathrm{H} \mathrm{NMR}\left(400 \mathrm{MHz}, \mathrm{CD}_{3} \mathrm{CN}\right)$ : $\delta=8.24\left(\right.$ br s, $\left.16 \mathrm{H}, \mathrm{H}_{\beta-p y r r}\right), 8.09\left(\mathrm{dd}, 8 \mathrm{H},{ }^{3} J_{\mathrm{H}, \mathrm{H}}=7.9 \mathrm{~Hz}\right.$ and ${ }^{4} J_{\mathrm{H}, \mathrm{H}}=1.7 \mathrm{~Hz}, \mathrm{H}_{o}$ out $), 7.95(\mathrm{br} \mathrm{d}, 8 \mathrm{H}$, ${ }^{3} J_{\mathrm{H}, \mathrm{H}}=7.9 \mathrm{~Hz}, \mathrm{H}_{m}$ in $), 7.83\left(\mathrm{dd}, 8 \mathrm{H},{ }^{3} J_{\mathrm{H}, \mathrm{H}}=7.4 \mathrm{~Hz}\right.$ and ${ }^{4} J_{\mathrm{H}, \mathrm{H}}=1.9 \mathrm{~Hz}, \mathrm{H}_{m}$ out $), 7.54\left(\mathrm{~d}, 8 \mathrm{H},{ }^{3} J_{\mathrm{H}, \mathrm{H}}=\right.$ $\left.2.0 \mathrm{~Hz}, \mathrm{H}^{5}\right), 7.43\left(\mathrm{~d}, 8 \mathrm{H},{ }^{3} J_{\mathrm{H}, \mathrm{H}}=2.0 \mathrm{~Hz}, \mathrm{H}^{4}\right), 7.39\left(\mathrm{dd}, 8 \mathrm{H},{ }^{3} J_{\mathrm{H}, \mathrm{H}}=7.4 \mathrm{~Hz}\right.$ and ${ }^{4} J_{\mathrm{H}, \mathrm{H}}=1.9 \mathrm{~Hz}, \mathrm{H}_{o}$ in), $5.78\left(\mathrm{~s}, 16 \mathrm{H}, \mathrm{CH}_{2}\right), 4.48\left(\mathrm{t}, 16 \mathrm{H},{ }^{3} J_{\mathrm{H}, \mathrm{H}}=6.9 \mathrm{~Hz}, \mathrm{H}_{a}\right), 2.05\left(\mathrm{~m}, 16 \mathrm{H}, \mathrm{H}_{b}\right), 1.55-1.45(\mathrm{~m}, 16 \mathrm{H}$, $\left.\mathrm{H}_{c}\right), 1.01 \mathrm{ppm}\left(\mathrm{t}, 24 \mathrm{H},{ }^{3} \mathrm{~J}_{\mathrm{H}, \mathrm{H}}=7.3 \mathrm{~Hz},-\mathrm{CH}_{3}\right) ;{ }^{13} \mathrm{C}\left\{{ }^{1} \mathrm{H}\right\} \mathrm{NMR}\left(150.9 \mathrm{MHz}, \mathrm{CD}_{3} \mathrm{CN}\right): \delta=184.1$ $\left(C_{N H C}\right), 149.9\left(C_{\alpha-p y r r}\right), 144.2\left(\mathrm{C}_{p}\right), 136.4\left(\mathrm{C}_{i}\right), 136.1\left(\mathrm{C}_{\text {o in }}\right), 134.3\left(\mathrm{C}_{\text {oout }}\right), 131.9\left(C_{\beta-p y r r}\right), 127.8\left(\mathrm{C}_{m}\right.$ out $), 126.4\left(\mathrm{C}_{m \text { in }}\right), 123.9\left(\mathrm{C}^{4}\right), 122.7\left(\mathrm{C}^{5}\right), 120.0\left(\mathrm{C}_{\text {meso }}\right), 55.5\left(\mathrm{CH}_{2}\right), 52.1\left(\mathrm{C}_{a}\right), 34.3\left(\mathrm{C}_{b}\right), 20.6\left(\mathrm{C}_{c}\right)$, 
$14.1 \mathrm{ppm}\left(\mathrm{CH}_{3}\right) ;{ }^{19} \mathrm{~F}\left\{{ }^{1} \mathrm{H}\right\} \mathrm{NMR}\left(376.5 \mathrm{MHz}, \mathrm{CD}_{3} \mathrm{CN}\right): \delta=-73.8 \mathrm{ppm}\left(\mathrm{d},{ }^{1} J_{\mathrm{F}, \mathrm{P}}=706.6 \mathrm{~Hz}\right) ;$ ${ }^{31} \mathrm{P}\left\{{ }^{1} \mathrm{H}\right\}$ NMR (162 MHz, $\left.\mathrm{CD}_{3} \mathrm{CN}\right): \delta=-144.7 \mathrm{ppm}$ (sept, ${ }^{1} J_{\mathrm{P}, \mathrm{F}}=706.6 \mathrm{~Hz}$ ); UV-Vis (DMSO): $\lambda_{\max }(\log \varepsilon)=424(5.71), 562(4.58), 604 \mathrm{~nm}$ (4.42); HRMS (ESI+): $m / z$ calcd for $\mathrm{C}_{152} \mathrm{H}_{152} \mathrm{~N}_{24} \mathrm{Zn}_{2} \mathrm{Au}_{4}{ }^{4+}\left[\mathrm{M}-4 \mathrm{PF}_{6}\right]^{4+}: 808.2473$; found: 808.2485 ; MS (ESI-): $m / z$ calcd for $\mathrm{PF}_{6}^{-}$: 144.96; found: 144.96 .

\section{RESULTS AND DISCUSSION}

Preparation of porphyrin-NHC precursors. Deprotonation of azolium salts with a base in a presence of metal ions is one of the most simple and efficient synthetic pathways to obtain NHC-metal complexes. ${ }^{46-48}$ For this purpose, we synthesized zinc(II) porphyrins with imidazolium groups on the para positions of the four meso positions (Scheme1) as porphyrin tetrakis(NHC) precursors. ${ }^{42}$ Rigid (1a and $\left.\mathbf{1 b}\right)$ and flexible (1c and 1d) porphyrin tetrakis(NHC) ligands were investigated. Rigid tetrakis(NHC) ligands $\mathbf{1 a}$ and $\mathbf{1} \mathbf{b}$ were obtained by directly attaching the NHC on the para positions of the four meso aryl groups. Flexible tetrakis-NHC ligands $\mathbf{1 c}$ and $\mathbf{1 d}$ were obtained by introducing methylene groups between the NHCs and the para positions of the four meso aryl groups. Long alkyl chains were used as peripheral NHC-wingtip groups in order to improve the solubility of the obtained complexes. Finally, zinc(II) was used as inner metal to protect the macrocycle from unwanted metalation reaction with silver(I) during the synthesis of cofacial porphyrin dimers. 
Scheme 1. Right: structures of porphyrins 1a-4a, 1b-4b, 1c-4c and 1d-4d. Left: synthesis of porphyrins $\mathbf{4 a - d}$.
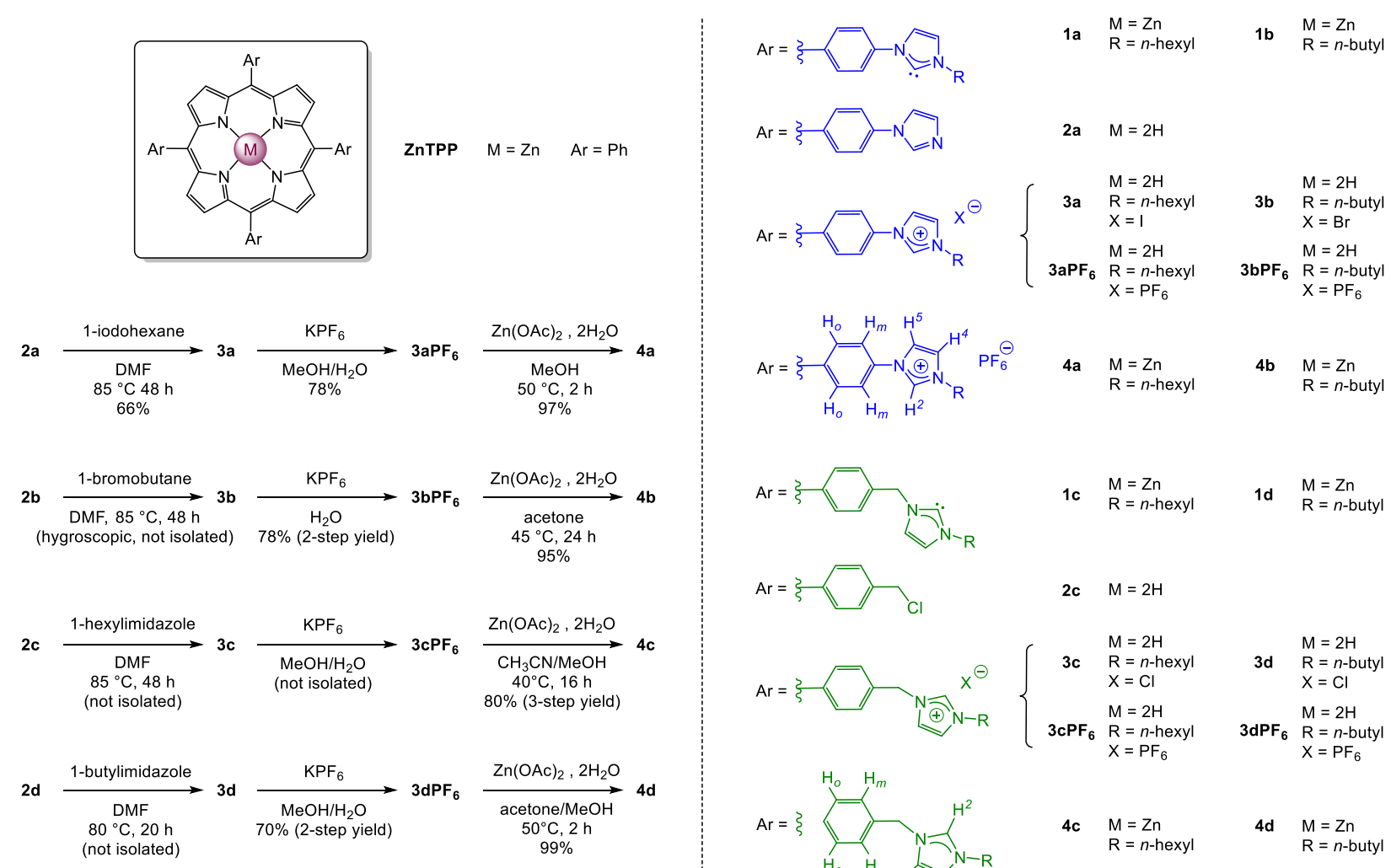

4a $\quad \begin{aligned} \mathrm{M} & =\mathrm{Zn} \\ \mathrm{R} & =n \text {-hexyl }\end{aligned}$

4b $\quad \begin{aligned} \mathrm{M} & =\mathrm{Zn} \\ \mathrm{R} & =n \text {-butyl }\end{aligned}$

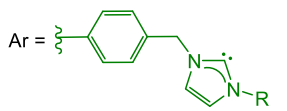

1c $\quad \mathrm{M}=\mathrm{Zn}$
$\mathrm{R}=n$-hexyl

1d $\quad \begin{aligned} & \mathrm{M}=\mathrm{Zn} \\ & \mathrm{R}=n \text {-butyl }\end{aligned}$

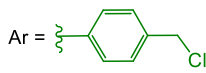

2c $\quad M=2 H$

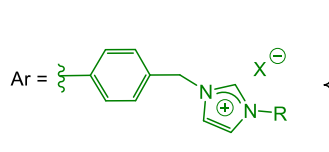

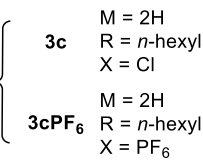

$\begin{array}{rl} & \mathrm{M}=2 \mathrm{H} \\ \text { 3d } & \mathrm{R}=n \text {-butyl } \\ & \mathrm{X}=\mathrm{Cl} \\ & \mathrm{M}=2 \mathrm{H} \\ \text { 3dPF }_{6} & \mathrm{R}=n \text {-butyl } \\ & \mathrm{X}=\mathrm{PF}_{6}\end{array}$

$\mathrm{Ar}=\xi$

4c $\quad \begin{array}{ll}M & =Z n \\ R & =n \text {-hexyl }\end{array}$

4d $\quad M=Z n$ $99 \%$

Porphyrins $\mathbf{4 a}$ and $\mathbf{4 b}$ was obtained in four steps starting from 4-(1H-imidazol-1yl)benzaldehyde (Scheme 1). The free-base meso 5,10,15,20-tetrakis(4'imidazolylphenyl)porphyrin $\mathbf{2 a}$ was prepared as previously reported. ${ }^{44}$ Then, alkylation of the four peripheral imidazole groups afforded porphyrin $\mathbf{3 a}$ and $\mathbf{3 b}$, which were directly engaged in anion metathesis reactions with $\mathrm{KPF}_{6}$. The obtained porphyrins $\mathbf{3 a P F}_{\mathbf{6}}$ and $\mathbf{3} \mathbf{b P F}_{\mathbf{6}}$ were metalated with $\mathrm{Zn}(\mathrm{OAc})_{2} \cdot 2 \mathrm{H}_{2} \mathrm{O}$ to obtain the two zinc(II) porphyrins $\mathbf{4 a}$ and $\mathbf{4 b}$, respectively. The ${ }^{1} \mathrm{H}$ NMR spectra of these porphyrins showed the expected deshielded signals of the imidazolium $\mathrm{H}^{2}$ protons 
at $\delta \sim 10 \mathrm{ppm}$ in DMSO- $d_{6}$. The signals of $\mathrm{PF}_{6}{ }^{-}$anions were observed at $\delta=-146.5 \mathrm{ppm}$ (septuplet) and $\delta=-71.1 \mathrm{ppm}$ (doublet) by ${ }^{31} \mathrm{P}\left\{{ }^{1} \mathrm{H}\right\}$ and ${ }^{19} \mathrm{~F}\left\{{ }^{1} \mathrm{H}\right\}$ NMR spectroscopy, respectively. The metalation of the porphyrin core with zinc(II) was confirmed by ${ }^{1} \mathrm{H}$ NMR spectroscopy, UV-Vis absorption spectroscopy and mass spectrometry. Porphyrins $\mathbf{4 b}$ and $\mathbf{4 c}$ were also prepared in four steps starting from 4-(chloromethyl)benzaldehyde (Scheme 1). The free-base meso 5,10,15,20tetrakis(4'-chloromethylphenyl)porphyrin $\mathbf{2 c}$ was prepared as previously reported. ${ }^{45}$ It was then reacted with 1-hexylimidazole and 1-butylimidazole to obtain the corresponding porphyrins $\mathbf{3 c}$ and $\mathbf{3 d}$, respectively. These compounds were directly engaged in the anion metathesis reaction with $\mathrm{KPF}_{6}$ to obtain the corresponding imidazolium salts $\mathbf{3} \mathbf{C P F} 6$ and $\mathbf{3 d P F}$. Finally, metalation reaction with $\mathrm{Zn}(\mathrm{OAc})_{2} \cdot 2 \mathrm{H}_{2} \mathrm{O}$ afforded the zinc(II) porphyrins $\mathbf{4} \mathbf{c}$ and $\mathbf{4 d}$. Their spectroscopic data are also in agreement with the proposed structures.

Preparation of porphyrin cages. Porphyrins 4a-d were used as tetrakis-NHC precursors for the synthesis of porphyrin cages (Scheme 2). NHCs are able to bind strongly to transition metal ions in low and high oxidation states. ${ }^{46-48}$ This is not advantageous to obtain coordination driven self-assembled supramolecular complexes, because reversible coordination bonds are needed to allow self-correction leading to the formation of thermodynamic compounds. However, $\mathrm{Ag}^{+}-\mathrm{C}_{\mathrm{NHC}}$ bonds are notable exceptions and are rather labile leading back to the formation of free NHCs and $\mathrm{Ag}^{+}$ions. Therefore, NHC ligands combined with $\mathrm{Ag}^{+}$ions behave like Werner-type compounds and are suitable building blocks to obtain coordination driven self-assembled supramolecular complexes. Moreover, the reversibility of the $\mathrm{Ag}^{+}-\mathrm{C}_{\mathrm{NHC}}$ bonds is routinely used to transmetalate NHCs from $\mathrm{Ag}^{+}$to other transition metal ions like $\mathrm{Au}^{+}{ }^{49}$ This strategy was applied for the synthesis of coordination-driven metallacycles and metallacages from poly-NHC ligands. ${ }^{50-53}$ 
The porphyrin cages $\left[\mathbf{A g}_{\mathbf{4}}(\mathbf{1} \mathbf{a}-\mathbf{d})_{2}\right]\left(\mathbf{P F}_{6}\right)_{4}$ were prepared by reacting porphyrins $\mathbf{4 a - d}$ with $\mathrm{Ag}_{2} \mathrm{O}$ in $\mathrm{CH}_{3} \mathrm{CN}$ for 24-48 hours in the dark (Scheme 2). The tetracationic nature of the four dimeric species $\left[\mathbf{A g}_{\mathbf{4}}(\mathbf{1 a - d})_{2}\right]^{\mathbf{4}+}$ was confirmed by high resolution mass spectrometry (ESI+) with excellent agreements between experimental isotopic profiles and calculated ones (see mass spectra in the Supporting Information). Then, NHC ligands of silver(I) complexes $\left[\mathbf{A g}_{\mathbf{4}}(\mathbf{1} \mathbf{a}-\mathbf{d})_{2}\right]\left(\mathbf{P F}_{6}\right)_{4}$ were easily transmetalated from $\mathrm{Ag}^{+}$to $\mathrm{Au}^{+}$by reacting porphyrin cages with $[\mathrm{AuCl}(\mathrm{tht})]($ tht $=$ tetrahydrothiophene) (Scheme 2) to obtain the corresponding gold(I) complexes [Au4(1ad)2 $\left.\mathbf{P}_{(\mathbf{P F}}\right)_{4}$. The expected molecular mass peaks of these complexes were observed with isotopically resolved profiles in good agreement with the calculated distributions (see for example the experimental and calculated mass spectra of the porphyrin cage $\left[\mathbf{A u}_{4}(\mathbf{1 a})_{2}\right]\left(\mathbf{P F}_{6}\right)_{4}$ in Figure 1).

Scheme 2. Synthesis of cofacial porphyrin dimers $\left[\mathbf{M}_{4}(\mathbf{1} \mathbf{a}-\mathbf{d})_{2}\right]\left(\mathbf{P F}_{6}\right)_{4}(\mathrm{M}=\mathrm{Ag}$ or $\mathrm{Au}, \mathrm{Hex}=n$ hexyl, $\mathrm{Bu}=n$-butyl, tht $=$ tetrahydrothiophene).
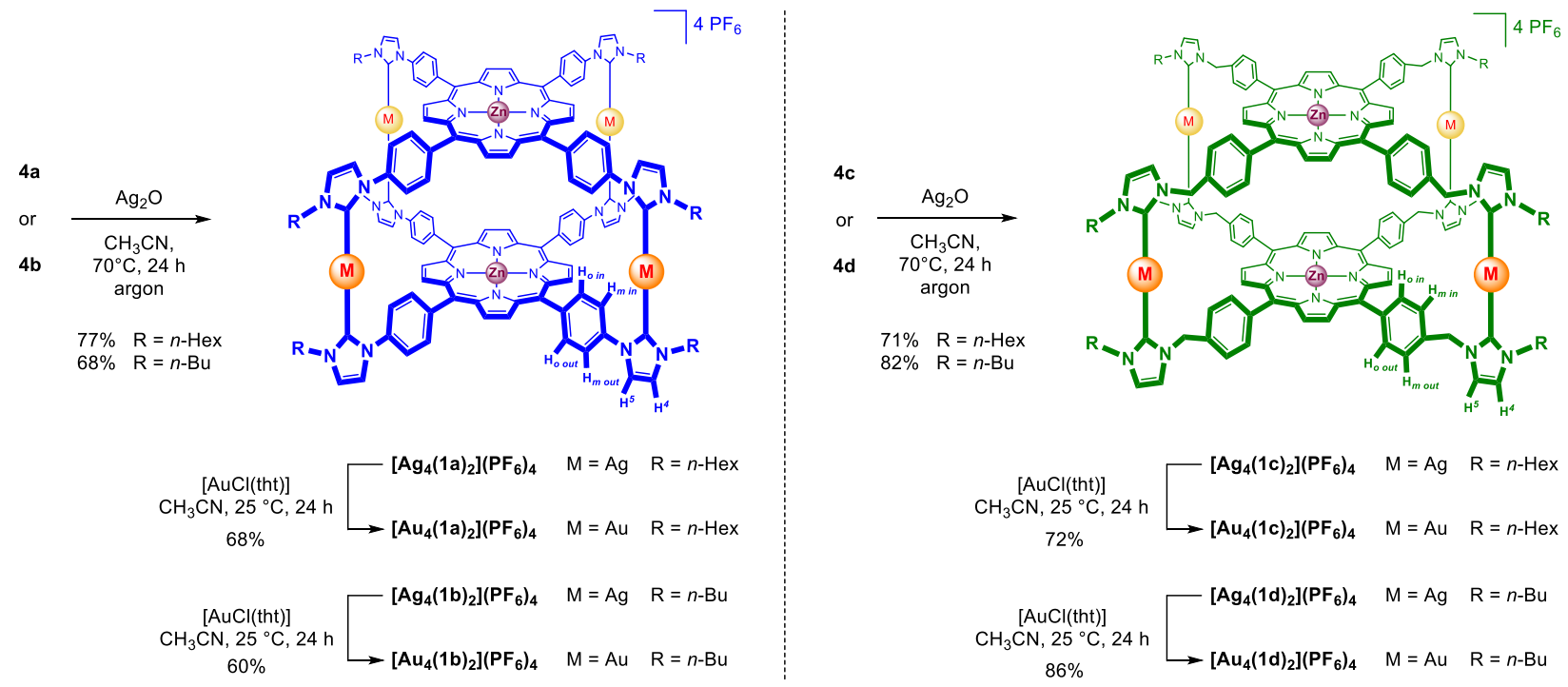
These porphyrin cages were characterized by ${ }^{1} \mathrm{H}$ and ${ }^{13} \mathrm{C}\left\{{ }^{1} \mathrm{H}\right\}$ NMR spectroscopy. The choice of the solvent (DMSO- $d_{6}, \mathrm{CD}_{3} \mathrm{CN}$ or $\mathrm{CD}_{2} \mathrm{Cl}_{2}$ ) is of crucial importance to obtain spectra with well resolved signals. As a representative example, the aromatic regions of the ${ }^{1} \mathrm{H}$ NMR spectra of the rigid porphyrin cages $\left[\mathbf{A g}_{4}\left(\mathbf{1 a}_{2}\right)_{2}\right]\left(\mathbf{P F}_{6}\right)_{4}$ and $\left[\mathbf{A u}_{4}\left(\mathbf{1}_{\mathbf{a}}\right)_{2}\right]\left(\mathbf{P F}_{6}\right)_{4}$ in DMSO-d $d_{6}$ are represented in Figure 1 and are consistent with a similar face-to-face orientation of the porphyrins and an average structure of $D_{4 h}$ symmetry. As illustrated in Figure 1a, the signals of the ortho $\left(\mathrm{H}_{o}\right)$ and meta $\left(\mathrm{H}_{m}\right)$ protons of the meso aryl groups of precursor $4 \mathbf{4}$ are observed as two doublets. In the ${ }^{1} \mathrm{H}$ NMR spectrum of the cofacial dimer $\left[\mathbf{A g}_{4}\left(\mathbf{1 a}_{\mathbf{a}}\right)_{2}\right]\left(\mathbf{P F}_{\mathbf{6}}\right)_{4}$ and $\left[\mathbf{A u}_{\mathbf{4}}\left(\mathbf{1 a}_{\mathbf{2}}\right)_{2}\right]\left(\mathbf{P F}_{\mathbf{6}}\right)_{4}$ represented in Figures $1 \mathrm{~b}$ and $1 \mathrm{c}$, respectively, four separated doublets are observed for ortho and meta protons suggesting that rotation of the meso aryl groups is restricted within the dimeric structures. As a consequence, different chemical environments are experienced by all meso aryl protons, namely $\mathrm{H}_{\text {o out }}, \mathrm{H}_{\text {m out }}, \mathrm{H}_{\text {o in }}$ and $\mathrm{H}_{\text {m in }}$ (see Scheme 2 and Figures $1 \mathrm{~b}$ and $1 \mathrm{c}$; attribution of the signals is based on 2D NMR experiments). The protons oriented inside the cofacial porphyrin dimer $\left(\mathrm{H}_{o}\right.$ in and $\mathrm{H}_{m}$ in) have upfield chemical shifts because of the shielding effect of the second porphyrin, whereas the protons oriented outside the cofacial porphyrin dimer $\left(\mathrm{H}_{\text {o out }}\right.$ and $\mathrm{H}_{m}$ out $)$ have chemical shifts rather similar to those observed for the monomeric porphyrin 4a. Similar observations could be made for the other porphyrin cages. In the ${ }^{13} \mathrm{C}\left\{{ }^{1} \mathrm{H}\right\}$ NMR spectra of gold(I) complexes [Aut 4 (1ad)2 2 (PF $)_{4}$, the signals of the NHC were observed as singlets at $\delta \sim 180-185 \mathrm{ppm}$ in good agreement with data reported in the literature. ${ }^{42,46-48}$ 

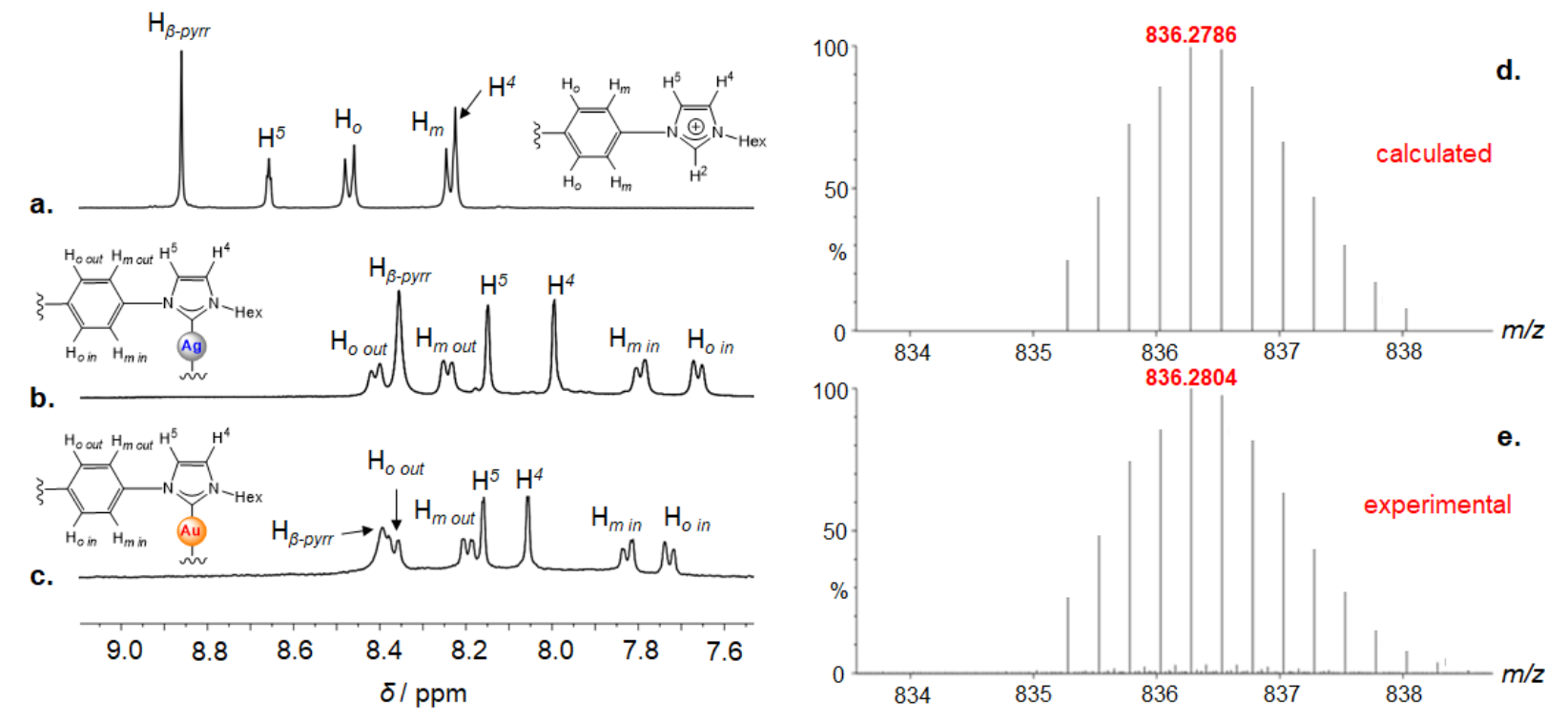

Figure 1 Left: stacked partial ${ }^{1} \mathrm{H}$ NMR (400 MHz) spectra in DMSO- $d_{6}$ of porphyrin 4a (a), and porphyrin cages $\left[\mathrm{Ag}_{4}\left(\mathbf{1 a}_{2}\right)_{2}\right]\left(\mathbf{P F}_{6}\right)_{4}$ (b) and $\left[\mathbf{A u}_{4}\left(\mathbf{1 a}_{2}\right)_{2}\right]\left(\mathbf{P F}_{6}\right)_{4}$ (c). Right: calculated (d) and experimental (e) isotopic distribution profiles of the tetracationic species $\mathrm{C}_{160} \mathrm{H}_{168} \mathrm{~N}_{24} \mathrm{Zn}_{2} \mathrm{Au}_{4}{ }^{4+}$, namely $\left[\mathrm{Au}_{4}(\mathbf{1 a})_{2}\right]^{4+}$.

Conformations of the porphyrin cages in solution. ${ }^{1} \mathrm{H}$ NMR and UV-visible absorption spectroscopies were used to determine conformations adopted by porphyrin cages in solution. We conducted these studies on gold(I) complexes which are more stable and less sensitive to daylight compared to the corresponding silver(I) complexes. ${ }^{42}$ Moreover, well-resolved ${ }^{1} \mathrm{H}$ NMR spectra were obtained for all gold(I) complexes. Porphyrins have characteristic UV-visible absorption spectra and changes observed in their absorption bands indicate modifications in porphyrins structure or, as it is the case here, in their environment and spatial organization. Indeed, $\mathrm{H}$ aggregates exhibit a Soret absorption band blue-shift with respect to the one of the monomer, corresponding to a face-to-face stacking of the monomeric species. In contrast, $J$-aggregates are 
edge-by-edge or side-to-side assemblies that produce a Soret absorption band red-shift with respect to the one of the monomer. ${ }^{54}$ Here, $\mathbf{Z n T P P}$ was taken as a reference compound and three solvents were used with increasing polarity in the order $\mathrm{CH}_{2} \mathrm{Cl}_{2}<\mathrm{CH}_{3} \mathrm{CN}<$ DMSO. In Figure 2 are gathered UV-visible absorption spectra of porphyrin cages $\left[\mathbf{A u}_{4}\left(\mathbf{1 a}_{2}\right)_{2}\right]\left(\mathbf{P F}_{6}\right)_{4}$ and $\left[\mathbf{A u}_{4}\left(\mathbf{1 c}_{2}\right)_{2}\right]\left(\mathbf{P F}_{6}\right)_{4}$ with $n$-hexyl NHC-wingtip groups in the range of wavelengths where the Soret absorption bands of porphyrins can be observed. Almost identical UV-visible spectra were obtained for porphyrin cages $\left[\mathbf{A u}_{4}(\mathbf{1})_{2}\right)_{2}\left(\mathbf{P F}_{6}\right)_{4}$ and $\left[\mathbf{A u}_{4}(\mathbf{1 d})_{2}\right]\left(\mathbf{P F}_{6}\right)_{4}$ with $n$-butyl NHC-wingtip groups (see the Supporting Information, Figure S128). As it is illustrated in Figure 2, Soret absorption bands of porphyrins belonging to cofacial porphyrin dimers $\left[\mathbf{A u}_{4}\left(\mathbf{1 a}_{\mathbf{2}}\right)_{2}\right]\left(\mathbf{P F}_{6}\right)_{4}$ and $\left[\mathbf{A u}_{4}\left(\mathbf{1}_{\mathbf{c}}\right)_{2}\right]\left(\mathbf{P F}_{6}\right)_{4}$ are blue-shifted with respect to the one of ZnTPP in all solvents. On the contrary, Q-bands of porphyrin cages are slightly red-shifted with respect to those of ZnTPP. These spectral evolutions are consistent with the proposed face-to-face orientation between the two porphyrins.
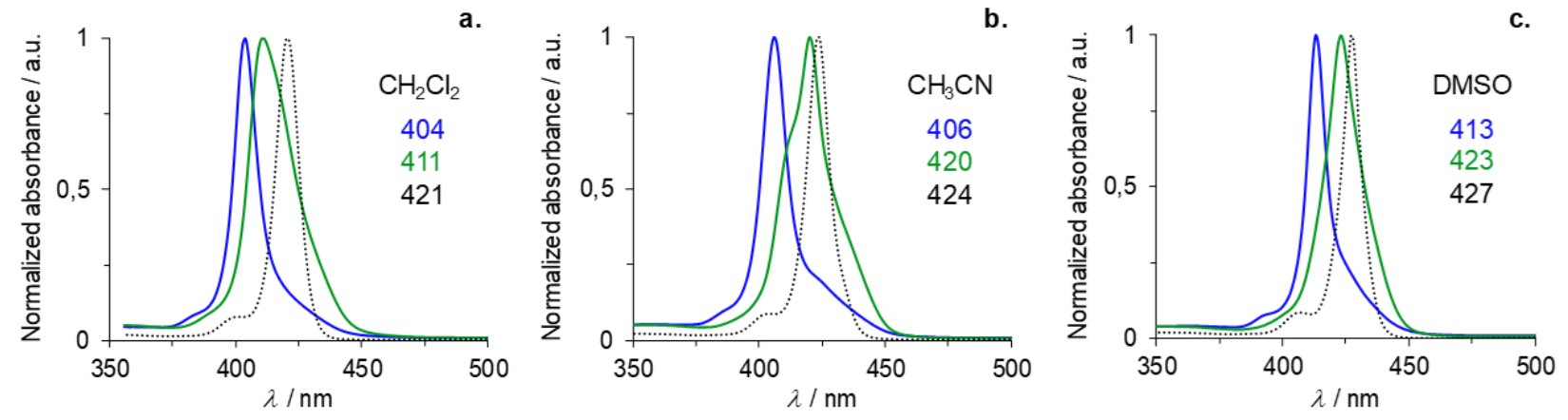

Figure 2. UV-Visible absorption spectra of ZnTPP (dotted black), and porphyrin cages $\left[\mathrm{Au}_{4}\left(\mathbf{1 a}_{2}\right)_{2}\right]\left(\mathbf{P F}_{6}\right)_{4}$ (blue) and $\left[\mathrm{Au}_{4}(\mathbf{1} \mathbf{c})_{2}\right]\left(\mathbf{P F}_{6}\right)_{4}$ (green) in $\mathrm{CH}_{2} \mathrm{Cl}_{2}$ (a), $\mathrm{CH}_{3} \mathrm{CN}$ (b) and DMSO (c). Values of $\lambda_{\max }$ of the Soret absorption bands are given. Normalized absorbance of the Soret absorption band at $\mathrm{A}=1.0$. 
The porphyrin cage $\left[\mathbf{A u}_{4}\left(\mathbf{1 a}_{2}\right)_{2}\right]\left(\mathbf{P F}_{\mathbf{6}}\right)_{4}$ has the most blue-shifted Soret absorption band with respect to the one of ZnTTP. It suggests that porphyrin cage incorporating the rigid phenyl groups between the porphyrin cores and the peripheral NHC ligands has probably the shortest porphyrinporphyrin distance. In $\mathrm{CH}_{2} \mathrm{Cl}_{2}$, the porphyrin cage $\left[\mathbf{A u}_{4}\left(\mathbf{1}_{\mathbf{a}}\right)_{2}\right]\left(\mathbf{P F}_{\mathbf{6}}\right)_{4}$ (Figure 2a, blue spectrum) exhibits a Soret absorption band with a maximum at $\lambda_{\max }=404 \mathrm{~nm}$ as compared to the Soret absorption band of the reference $\mathbf{Z n T T P}\left(\lambda_{\max }=421 \mathrm{~nm}, \Delta \lambda_{\max }=17 \mathrm{~nm}\right.$, black dotted spectrum in Figure 2a). A weak shoulder around $\sim 420-450 \mathrm{~nm}$ is also noticeable. In more polar and coordinating solvents like $\mathrm{CH}_{3} \mathrm{CN}$ and DMSO, the shape of the Soret absorption band of the porphyrin cage $\left[\mathbf{A u}_{4}\left(\mathbf{1 a}_{2}\right)_{2}\right]\left(\mathbf{P F}_{6}\right)_{4}$ remains constant (Figures $2 \mathrm{~b}$ and $2 \mathrm{c}$, blue spectra). The hypsochromic shifts observed for the Soret absorption bands with respect to the one of ZnTPP are also in the same range ( $\Delta \lambda_{\max }=18$ and $14 \mathrm{~nm}$ in $\mathrm{CH}_{3} \mathrm{CN}$ and DMSO, respectively) suggesting that the porphyrin-porphyrin distances are rather similar in these three solvents. This may be explained by the rigidity of the porphyrin cages incorporating the phenyl groups between the porphyrin cores and the peripheral NHC ligands.

$\mathrm{UV}$-visible absorption spectrum of porphyrin cage $\left[\mathbf{A u}_{4}\left(\mathbf{1} \mathbf{c}_{2}\right]\left(\mathbf{P F}_{6}\right)_{4}\right.$ in $\mathrm{CH}_{2} \mathrm{Cl}_{2}$ exhibits a Soret absorption band with a maximum at $\lambda_{\max }=411 \mathrm{~nm}$ (Figure 2a, green spectrum). The observed hypsochromic shift $\left(\Delta \lambda_{\max }=10 \mathrm{~nm}\right)$ of the Soret absorption bands with respect to the one of ZnTPP is also consistent with a face-to-face orientation between the two porphyrins. However, the blue-shift observed for $\left[\mathbf{A u}_{4}\left(\mathbf{1}_{\mathbf{c}}\right)_{2}\right]\left(\mathbf{P F}_{6}\right)_{4}$ is less important $(10 \mathrm{~nm}$ vs $17 \mathrm{~nm})$ compared to the one observed for $\left[\mathbf{A u}_{4}\left(\mathbf{1 a}_{\mathbf{a}}\right)_{2}\right]\left(\mathbf{P F}_{6}\right)_{4}$. This may be attributed to a greater porphyrinporphyrin distance decreasing excitonic coupling between the two porphyrins. The Soret absorption bands of this porphyrin cage $\left[\mathbf{A u}_{4}\left(\mathbf{1}_{\mathbf{c}}\right)_{2}\right]\left(\mathbf{P F}_{6}\right)_{4}$ is also very broad with a full width at half maximum of $\Delta \lambda=22 \mathrm{~nm}(\Delta \lambda=11 \mathrm{~nm}$ for $\mathbf{Z n T P P}$ and for the rigid porphyrin cage 
$\left[\mathrm{Au}_{4}\left(\mathbf{1 c}_{2}\right]\left(\mathbf{P F}_{6}\right)_{4}\right)$. This is also the case in polar solvents like $\mathrm{CH}_{3} \mathrm{CN}$ and DMSO. Indeed, shoulders can be clearly observed in the Soret absorption band of $\left[\mathbf{A u}_{4}(\mathbf{1 c})_{2}\right]\left(\mathbf{P F}_{6}\right)_{4}$ in $\mathrm{CH}_{3} \mathrm{CN}$. These spectral features are attributed to the presence of several cofacial conformations in solution due to the incorporation of the more flexible meso benzyl groups. ${ }^{55}$ It is also remarkable that Soret absorption bands of the flexible porphyrin cage $\left[\mathbf{A u}_{4}\left(\mathbf{1 c}_{\mathbf{c}} \mathbf{2}_{2}\right]\left(\mathbf{P F}_{6}\right)_{4}\right.$ is blue-shifted by only $\Delta \lambda_{\max }=$ $4 \mathrm{~nm}$ with respect to the one of $\mathbf{Z n T P P}$ in polar solvents like $\mathrm{CH}_{3} \mathrm{CN}$ and DMSO, while this hypsochromic shift is more important for $\left[\mathbf{A u}_{4}\left(\mathbf{1 a}_{2}\right)_{2}\right]\left(\mathbf{P F}_{\mathbf{6}}\right)_{4}\left(\Delta \lambda_{\max }=18\right.$ and $13 \mathrm{~nm}$ in $\mathrm{CH}_{3} \mathrm{CN}$ and DMSO, respectively). This observation suggests that the porphyrin-porphyrin distance of the flexible porphyrin cage $\left[\mathbf{A u}_{4}\left(\mathbf{1}_{\mathbf{c}}\right)_{2}\right]\left(\mathbf{P F}_{6}\right)_{4}$ is greater in $\mathrm{CH}_{3} \mathrm{CN}$ and DMSO ("open" conformation) than in $\mathrm{CH}_{2} \mathrm{Cl}_{2}$ ("closed" conformation). It contrasts with the fact that the porphyrin-porphyrin distance of the rigid porphyrin cage $\left[\mathbf{A u}_{4}\left(\mathbf{1 a}_{2}\right)_{2}\right]\left(\mathbf{P F}_{6}\right)_{4}$ remains relatively the same whatever the nature of the solvent.

Conformation of porphyrin cages was also investigated by ${ }^{1} \mathrm{H}$ NMR spectroscopy. Aromatic regions of ${ }^{1} \mathrm{H}$ NMR spectra in $\mathrm{CD}_{3} \mathrm{CN}$ and $\mathrm{CD}_{2} \mathrm{Cl}_{2}$ of porphyrin cages [Au4(1a$\mathbf{d}_{2} \mathbf{2}_{\mathbf{2}}\left(\mathbf{P F}_{6}\right)_{4}$ are represented in Figure $3 .{ }^{1} \mathrm{H}$ NMR spectra of both porphyrin cages $\left[\mathbf{A u}_{4}\left(\mathbf{1 a}_{2}\right)_{2}\right]\left(\mathbf{P F}_{6}\right)_{4}$ and $\left[\mathbf{A u}_{4}(\mathbf{1} \mathbf{b})_{2}\right]\left(\mathbf{P F}_{6}\right)_{4}$ with $n$-hexyl and $n$-butyl NHC-wingtip groups, respectively, are very similar in $\mathrm{CD}_{3} \mathrm{CN}$ (Figures 3a vs. 3c) and in $\mathrm{CD}_{2} \mathrm{Cl}_{2}$ (Figures 3b vs. 3d). Therefore, the nature of the peripheral $n$-alkyl chains do not play a significant role on the conformation of the rigid porphyrin cages $\left[\mathbf{A u}_{4}\left(\mathbf{1 a}_{2}\right)_{2}\right]\left(\mathbf{P F}_{6}\right)_{4}$ and $\left[\mathbf{A u}_{4}\left(\mathbf{1 b}_{2}\right)_{2}\right]\left(\mathbf{P F}_{6}\right)_{4}$ in solution. These two cages also adopt similar conformations in $\mathrm{CD}_{3} \mathrm{CN}$ and $\mathrm{CD}_{2} \mathrm{Cl}_{2}$ since their ${ }^{1} \mathrm{H}$ NMR spectra are similar in both solvents (see

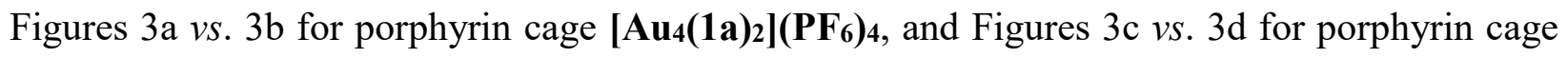
[Au4(1) $\left.\left.\mathbf{A b}_{2}\right]\left(\mathbf{P F}_{6}\right)_{4}\right)$. The singlets due to $\beta$-pyrrolic protons are in the same range in $\mathrm{CD}_{3} \mathrm{CN}(\delta=$ $8.58 \mathrm{ppm})$ and in $\mathrm{CD}_{2} \mathrm{Cl}_{2}(\delta=8.66 \mathrm{ppm})$ showing that porphyrin-porphyrin distances are similar 
in both solvents, in agreement with observations made by UV-visible absorption spectroscopy. Again, this can be explained by the rigidity of the porphyrin cages $\left[\mathbf{A u}_{4}\left(\mathbf{1 a}_{\mathbf{a}} \mathbf{2}_{\mathbf{2}} \mathbf{P}_{\mathbf{P}} \mathbf{P F}_{\mathbf{4}}\right.\right.$ and [Au4(1) $\left.\mathbf{A b}_{2}\right]\left(\mathbf{P F}_{6}\right)_{4}$ with meso phenyl groups between porphyrin cores and peripheral NHCs.
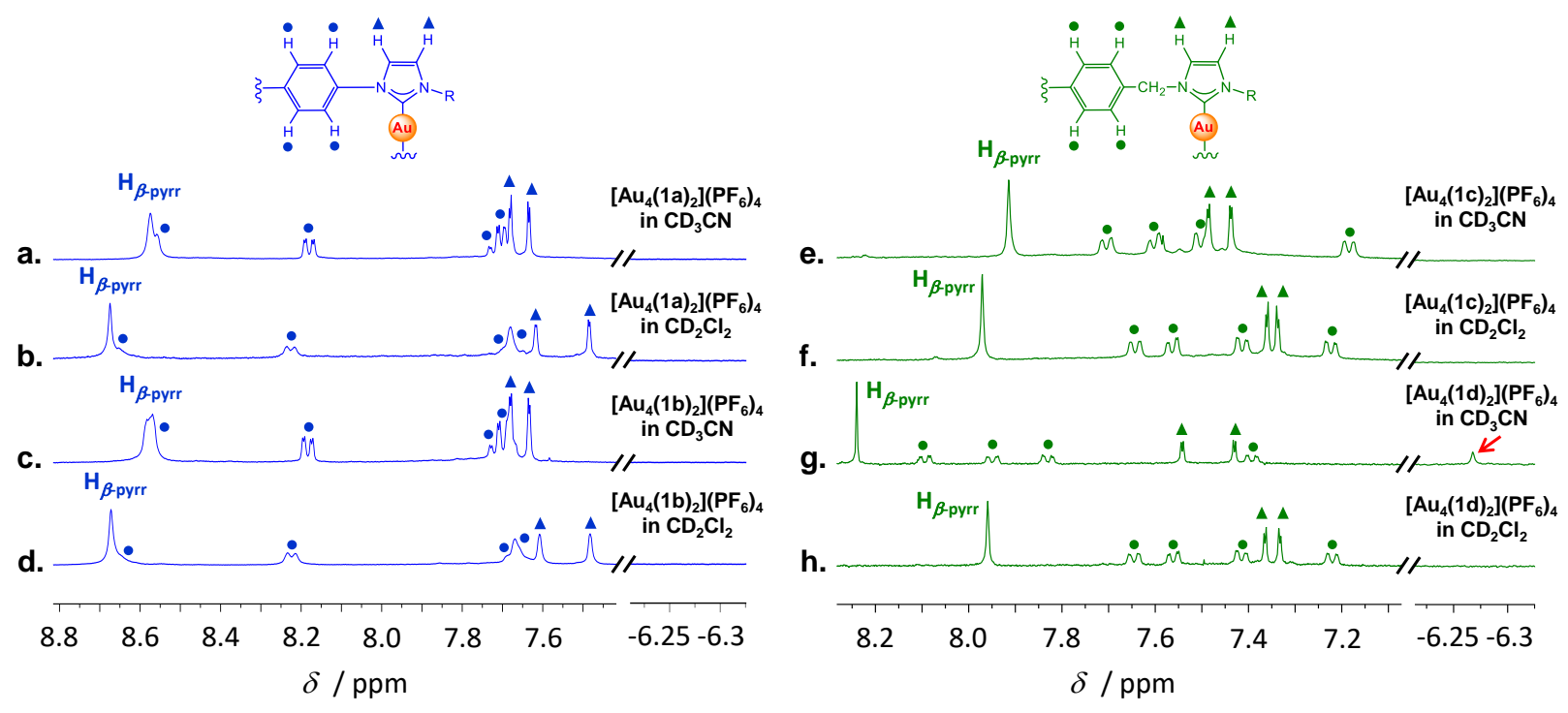

Figure 3. ${ }^{1} \mathrm{H}$ NMR spectra $(400 \mathrm{MHz})$ of porphyrin cages $\left[\mathbf{A u}_{4}(\mathbf{1 a}-\mathbf{d})_{2}\right]\left(\mathbf{P F}_{6}\right)_{4}$ in $\mathrm{CD}_{3} \mathrm{CN}$ and $\mathrm{CD}_{2} \mathrm{Cl}_{2}\left(\bullet=\right.$ signals of meso aryl protons ; $\mathbf{\Lambda}=$ signals of $\mathrm{H}^{4}$ and $\mathrm{H}^{5}, \mathrm{R}=n$-hexyl or $n$-butyl groups $)$.

Surprising results were obtained when considering more flexible porphyrin cages $\left[\mathbf{A u}_{4}\left(\mathbf{1 c}_{2}\right)_{2} \mathbf{P F}_{6}\right)_{4}$ and $\left.\mathbf{A u}_{4}\left(\mathbf{1 d}_{2}\right)_{2} \mathbf{P F}_{6}\right)_{4}$ with meso benzyl groups between porphyrin cores and peripheral NHCs. The flexible porphyrin cage $\left[\mathbf{A u}_{4}\left(\mathbf{1 c}_{\mathbf{2}}\right]\left(\mathbf{P F}_{\mathbf{6}}\right)_{4}\right.$ with $n$-hexyl NHC-wingtip groups adopts similar conformations in $\mathrm{CD}_{3} \mathrm{CN}$ and $\mathrm{CD}_{2} \mathrm{Cl}_{2}$ since ${ }^{1} \mathrm{H}$ NMR spectra are similar in both solvents (see Figures 3e vs. 3f). The singlets due to $\beta$-pyrrolic protons are in the same range in $\mathrm{CD}_{3} \mathrm{CN}(\delta=7.91 \mathrm{ppm})$ and in $\mathrm{CD}_{2} \mathrm{Cl}_{2}(\delta=7.97 \mathrm{ppm})$ showing that porphyrin-porphyrin distances 
are similar in both solvents. Interestingly, a striking difference was observed when considering the porphyrin cage $\left[\mathbf{A u}_{4}(\mathbf{1 d})_{2}\right]\left(\mathbf{P F}_{6}\right)_{4}$ with $n$-butyl NHC-wingtip groups in $\mathrm{CD}_{3} \mathrm{CN}$ (Figure $3 \mathrm{~g}$ ) and in $\mathrm{CD}_{2} \mathrm{Cl}_{2}$ (Figure 3h). In $\mathrm{CD}_{2} \mathrm{Cl}_{2}$, the porphyrin cages $\left[\mathbf{A u}_{4}\left(\mathbf{1}_{\mathbf{c}}\right)_{2}\right]\left(\mathbf{P F}_{6}\right)_{4}$ and $\left[\mathbf{A u}_{4}(\mathbf{1 d})_{2}\right]\left(\mathbf{P F}_{6}\right)_{4}$ adopt the same conformation because the aromatic region of their ${ }^{1} \mathrm{H}$ NMR spectra are very similar (see Figures $3 \mathrm{f} v s .3 \mathrm{~h}$ ). It corresponds to the "closed" conformation observed by UV-visible spectroscopy in $\mathrm{CH}_{2} \mathrm{Cl}_{2}$. Surprisingly, conformation of the porphyrin cage $\left[\mathrm{Au}_{4}\left(\mathbf{1 d}_{2}\right)_{2}\right]\left(\mathbf{P F}_{6}\right)_{4}$ in $\mathrm{CD}_{3} \mathrm{CN}$ is different from that of the porphyrin cage $\left[\mathbf{A u}_{4}\left(\mathbf{1}_{\mathbf{c}}\right)_{2}\right]\left(\mathbf{P F}_{6}\right)_{4}$ in the same solvent (see Figures $3 \mathrm{~g} v s .3 \mathrm{e})$. All signals of the aromatic region are significantly shifted downfield ( $\delta=8.24$ ppm for the singlet due to $\beta$-pyrrolic protons). This spectral evolution may be attributed to a greater porphyrin-porphyrin distance in the case of the porphyrin cage $\left[\mathbf{A u}_{4}\left(\mathbf{1 d}_{2}\right]\left(\mathbf{P F}_{6}\right)_{4}\right.$. In the same time, a high upfield shifted signal is observed at $\delta=-6.27 \mathrm{ppm}$ (Figure 3g). This signal was only observed in the case of the porphyrin cage $\left[\mathrm{Au}_{4}\left(\mathbf{1 d}_{\mathbf{1}}\right)_{2}\right]\left(\mathbf{P F}_{\mathbf{6}}\right)_{4}$ in $\mathrm{CD}_{3} \mathrm{CN}$ as it is represented in Figure 3. Encapsulation of molecular species between the two porphyrins may explain the observed "open" conformation and the greater porphyrin-porphyrin distance in this case. This point is discussed in the next paragraph.

Encapsulation Properties. If there is enough space between the two porphyrins, porphyrin cages can encapsulate guest molecules and, as a consequence, protons of the encapsulated molecules are shielded by the ring currents of the two porphyrins. As there is no upfield signal in the ${ }^{1} \mathrm{H}$ NMR spectra of the rigid porphyrin cages $\left[\mathbf{A u}_{4}\left(\mathbf{1 a}_{2}\right)_{2}\right]\left(\mathbf{P F}_{\mathbf{6}}\right)_{4}$ and $\left[\mathbf{A u}_{4}\left(\mathbf{1 b}_{\mathbf{2}}\right)_{\mathbf{2}}\right]\left(\mathbf{P F}_{\mathbf{6}}\right)_{4}$ in $\mathrm{CD}_{3} \mathrm{CN}$ and $\mathrm{CD}_{2} \mathrm{Cl}_{2}$, the presence of guest molecules like solvent or $\mathrm{H}_{2} \mathrm{O}$ between the two porphyrins is very unlikely (Figure 3a-d). By contrast, an upfield singlet at $\delta=-6.27 \mathrm{ppm}$ was observed in the ${ }^{1} \mathrm{H}$ NMR spectrum of the flexible porphyrin cage $\left[\mathbf{A u}_{4}(\mathbf{1 d})_{2}\right]\left(\mathbf{P F}_{6}\right)_{4}$ in $\mathrm{CD}_{3} \mathrm{CN}$ (see Figure $3 \mathrm{~g}$ ). ${ }^{1} \mathrm{H}$ 2D diffusion-ordered spectroscopy (DOSY) NMR showed that this upfield signal and signals due 
to the porphyrin cage have the same diffusion coefficient indicating that they diffuse as a single entity (Figure 4a). These data strongly suggest the formation of a host-guest system between the porphyrin cage $\left[\mathbf{A u}_{4}(\mathbf{1 d})_{2}\right]\left(\mathbf{P F}_{6}\right)_{4}$ and encapsulated molecular species. Thus, the upfield singlet at $\delta=-6.27 \mathrm{ppm}$ may be attributed to encapsulated $\mathrm{CH}_{3} \mathrm{CN}$ (solvent used for the synthesis of porphyrin cages) or $\mathrm{H}_{2} \mathrm{O}$ molecules (present in $\mathrm{CD}_{3} \mathrm{CN}$ or generated in the course of the deprotonation of imidazolium salts by $\mathrm{Ag}_{2} \mathrm{O}$ ). Since no correlation with any carbon could be observed by ${ }^{1} \mathrm{H}-{ }^{13} \mathrm{C}\left\{{ }^{1} \mathrm{H}\right\}$ HSQC NMR spectroscopy, the signal at $\delta=-6.27 \mathrm{ppm}$ is not due to the methyl group of $\mathrm{CH}_{3} \mathrm{CN}$. Moreover, it was observed even if the synthesis of the cages was performed in $\mathrm{CD}_{3} \mathrm{CN}$. Hence, it may be assumed that this upfield signal is due to protons of encapsulated $\mathrm{H}_{2} \mathrm{O}$ molecules, although it was not possible to observe them by mass spectrometry. The most convincing evidence arises from the NMR experiment where $\mathrm{H}_{2} \mathrm{O}$ is added in excess to a solution of the porphyrin-cage $\left[\mathrm{Au}_{4}\left(\mathbf{1}_{\mathbf{c}}\right)_{2}\right]\left(\mathbf{P F}_{6}\right)_{4}$ with $n$-hexyl NHC-wingtip groups in $\mathrm{CD}_{3} \mathrm{CN}$. Initially, this porphyrin cage is in its "closed" conformation in $\mathrm{CD}_{3} \mathrm{CN}$ at a concentration in the millimolar range used for ${ }^{1} \mathrm{H}$ NMR spectroscopy and no high upfield signal could be observed (Figure $4 b)$. Upon addition of $\mathrm{H}_{2} \mathrm{O}(10 \% \mathrm{v} / \mathrm{v})$ to a solution of the porphyrin cage $\left[\mathbf{A u}_{4}(\mathbf{1} \mathbf{c})_{2}\right]\left(\mathbf{P F}_{6}\right)_{4}$ in $\mathrm{CD}_{3} \mathrm{CN}$, a new set of signals is observed by ${ }^{1} \mathrm{H}$ NMR spectroscopy (Figure 4c). These new signals are very similar to those observed in the ${ }^{1} \mathrm{H}$ NMR spectrum of the porphyrin cage $\left[\mathrm{Au}_{4}(\mathbf{1 d})_{2}\right]\left(\mathbf{P F}_{6}\right)_{4}$ in $\mathrm{CD}_{3} \mathrm{CN}$ (Figure 4d). Moreover, a new high upfield signal is observed at $\delta=$ $-6.22 \mathrm{ppm}$ and this signal is attributed to encapsulated $\mathrm{H}_{2} \mathrm{O}$ molecules (Figure 4c, red arrow). Integration values are above $2 \mathrm{H}(\sim 2.5-3 \mathrm{H})$ and suggest that probably two $\mathrm{H}_{2} \mathrm{O}$ molecules are axially coordinated to zinc(II) porphyrins (one $\mathrm{H}_{2} \mathrm{O}$ per zinc(II) ion) and directed inside the cavity. Indeed, many ligands and molecules show a preference for binding inside the cavity of porphyrin cages. ${ }^{56-59}$ The $\mathrm{H}_{2} \mathrm{O}$ proton signals appear as singlets because of the quick dynamic behavior on 
the ${ }^{1} \mathrm{H}$ NMR timescale. Both signals of free $\mathrm{H}_{2} \mathrm{O}$ and encapsulated $\mathrm{H}_{2} \mathrm{O}$ are independently observed as sharp signals. This indicates that the binding and release of $\mathrm{H}_{2} \mathrm{O}$ at room temperature is slower than the NMR timescale. Interestingly, we also observed that no (or very weak) sign of $\mathrm{D} / \mathrm{H}$ exchange was observed upon the addition of $\mathrm{D}_{2} \mathrm{O}$ to a solution of $\left[\mathrm{Au}_{4}(\mathbf{1 d})_{2}\right]\left(\mathbf{P F}_{6}\right)_{4}$ in $\mathrm{CD}_{3} \mathrm{CN}$. We presume that inner $\mathrm{H}_{2} \mathrm{O}$ molecules surrounded by the porphyrin cage $\left[\mathbf{A u}_{4}(\mathbf{1 d})_{2}\right]^{4+}$ are protected from $\mathrm{D} / \mathrm{H}$ exchange with outer $\mathrm{D}_{2} \mathrm{O}$ molecules. This observation is in line with the fact that, in some cases, chemical stability of encapsulated molecular species is remarkably improved within hosts. For example, Stoddart and coworkers recently reported the high chemical stability of a freebase porphyrin encapsulated within an X-shaped cyclophane receptor: protonation, $\mathrm{D} / \mathrm{H}$ exchange and solvolysis of the encapsulated free-base porphyrin are blocked by the cyclophane host. ${ }^{60}$
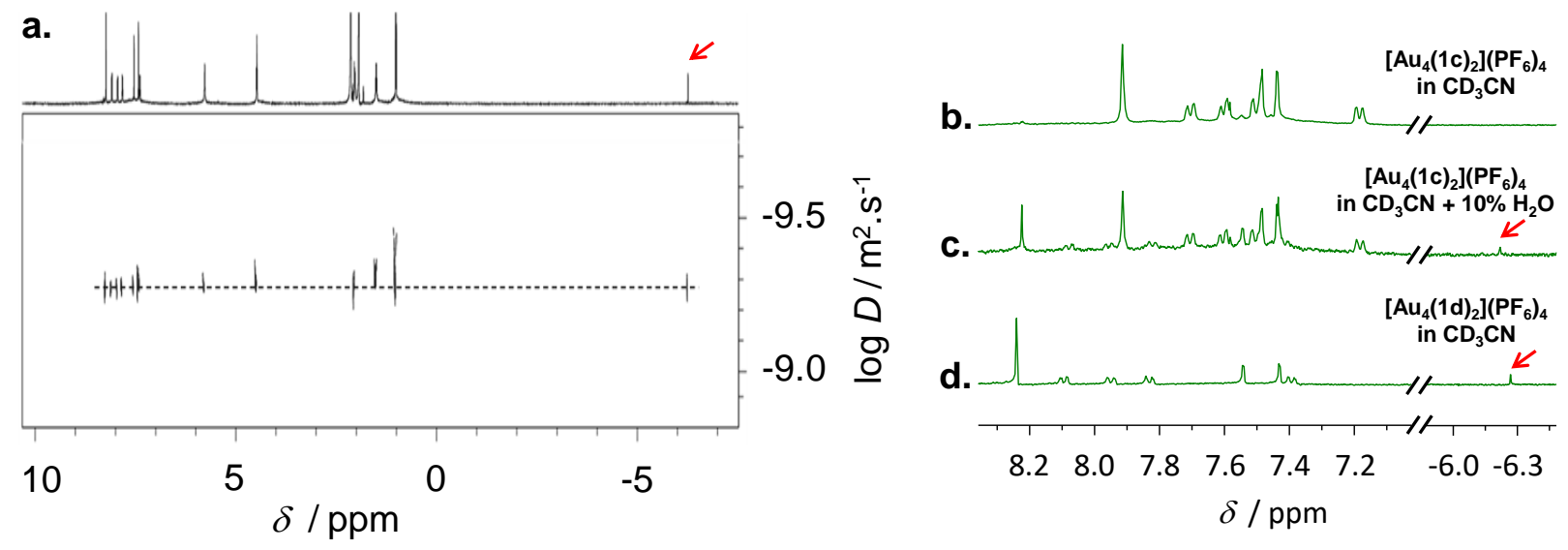

Figure 4. ${ }^{1} \mathrm{H} 2 \mathrm{D}$ DOSY NMR spectrum $(600 \mathrm{MHz})$ of porphyrin cage $\left[\mathbf{A u}_{4}(\mathbf{1 d})_{2}\right]\left(\mathbf{P F}_{\mathbf{6}}\right)_{4}$ in $\mathrm{CD}_{3} \mathrm{CN}$ (a). ${ }^{1} \mathrm{H}$ NMR spectra (400 MHz) of porphyrin cage $\left[\mathbf{A u}_{4}(\mathbf{1 c})_{2}\right]\left(\mathbf{P F}_{6}\right)_{4}$ in $\mathrm{CD}_{3} \mathrm{CN}$ (b) and in $\mathrm{CD}_{3} \mathrm{CN}$ $+10 \% \mathrm{H}_{2} \mathrm{O}(v / v)(\mathrm{c}) .{ }^{1} \mathrm{H}$ NMR spectrum $(400 \mathrm{MHz})$ of porphyrin cage $\left[\mathbf{A u}_{4}(\mathbf{1 d})_{2}\right]\left(\mathbf{P F}_{6}\right)_{4}$ in $\mathrm{CD}_{3} \mathrm{CN}$ (d). 
When comparing the two flexible porphyrin cages $\left[\mathbf{A u}_{4}\left(\mathbf{1 c}_{\mathbf{2}}\right]\left(\mathbf{P F}_{\mathbf{6}}\right)_{\mathbf{4}}\right.$ and $\left[\mathbf{A u}_{\mathbf{4}}(\mathbf{1 d})_{2}\right]\left(\mathbf{P F}_{\mathbf{6}}\right)_{4}$, it is remarkable to note that the length of the peripheral akyl chains ( $n$-hexyl vs. $n$-butyl) dramatically modifies the encapsulating properties. The porphyrin cage $\left[\mathbf{A u}_{4}(\mathbf{1 d})_{2} \mathbf{C}_{(\mathbf{P F}}\right)_{4}$ with peripheral $n$ butyl groups has a high affinity for $\mathrm{H}_{2} \mathrm{O}$ in $\mathrm{CD}_{3} \mathrm{CN}$ and it was not possible to observe the "closed" conformation (i.e. without $\mathrm{H}_{2} \mathrm{O}$ molecules): the amount of $\mathrm{H}_{2} \mathrm{O}$ present in commercially available $\mathrm{CD}_{3} \mathrm{CN}$ is sufficient and it is not necessary to add more $\mathrm{H}_{2} \mathrm{O}$ in the NMR sample. On the contrary, the "closed" conformation of the porphyrin cage $\left[\mathbf{A u}_{4}(\mathbf{1 c})_{2}\right]\left(\mathbf{P F}_{6}\right)_{4}$ with peripheral $n$-hexyl groups is the only conformation which is observed in $\mathrm{CD}_{3} \mathrm{CN}$. The "open" conformation encapsulating $\mathrm{H}_{2} \mathrm{O}$ molecules is observed when a large amount of $\mathrm{H}_{2} \mathrm{O}$ is added in the NMR sample. Thus, it is obvious that the length of the peripheral alkyl chains also plays an important role in the encapsulation of $\mathrm{H}_{2} \mathrm{O}$.

Density Functional Theory (DFT) calculations were performed to optimize the geometries of the rigid and flexible porphyrin cages, namely $\left[\mathbf{A u}_{4}(\mathbf{1 a} / \mathbf{b})_{2}\right]^{4+}$ and $\left[\mathbf{A u}_{4}(\mathbf{1 c} / \mathbf{d})_{2}\right]^{4+}$, in view of comparing their porphyrin-porphyrin distances (see the Supporting Information). Long alkyl chains are replaced by methyl groups to construct model systems and shorten computing time. Investigated gold(I) complexes are displayed in Figure 5. The calculated $\mathrm{Au}^{+}-\mathrm{C}_{\mathrm{NHC}}$ distances of 2.05-2.06 $\AA$ are in agreement with distances measured in X-ray crystal structures of linear bis(NHC) gold(I) complexes. ${ }^{50-52}$ Porphyrin cages $\left[\mathbf{A u}_{4}(\mathbf{1} \mathbf{a} / \mathbf{b})_{2}\right]^{4+}$ are considered without guest molecule between the two porphyrins as guest inclusion was not observed in the conditions used for this study (Figure 5a). The two porphyrins have a face-to-face orientation, but are not completely flat and adopt a slight out-of-plane saddle-shaped distortion. For this complex, the calculated $\mathrm{Zn}-\mathrm{Zn}$ distance is $\sim 5.53 \AA$. DFT calculations were further performed to explore the flexible porphyrin cages $\left[\mathbf{A u} \mathbf{4}(\mathbf{1} \mathbf{c} / \mathbf{d})_{2}\right]^{4+}$. In a first approach, no guest was included as it was 
experimentally observed in dichloromethane (Figure 5b). Interestingly, incorporation of the flexible benzyl group between the porphyrin cores and the peripheral NHCs allows the porphyrinporphyrin distance to be shorter. Indeed, the calculated $\mathrm{Zn}-\mathrm{Zn}$ distance is $\sim 3.82 \AA$. The two porphyrins also adopt a stronger out-of-plane saddle-shaped distortion. Then, we considered the inclusion of two $\mathrm{H}_{2} \mathrm{O}$ molecules within the flexible porphyrin cages $\left[\mathrm{Au}_{4}(\mathbf{1} \mathbf{c} / \mathbf{d})_{2}\right]^{4+}$ (Figure $5 \mathrm{c}$ ). Both $\mathrm{H}_{2} \mathrm{O}$ molecules are coordinated to zinc(II) porphyrins and linked together by a hydrogen bond, as it was previously observed and/or calculated for other $\mathrm{H}_{2} \mathrm{O}$ dimers encapsulated within discrete 3D-molecular hosts such as superphane ${ }^{61}$ or fullerene $\mathrm{C}_{70} .{ }^{62}$ The calculated $\mathrm{Zn}$ - Zn distance of $7.44 \AA$ is significantly longer compared to the one calculated for $\left[\mathbf{A u}_{4}(\mathbf{1} \mathbf{a} / \mathbf{b})_{2}\right]^{4+}(\sim+1.9 \AA)$ and in agreement with the less remarkable blueshift of the Soret absorption band observed by UVVisible absorption spectroscopy.

a.
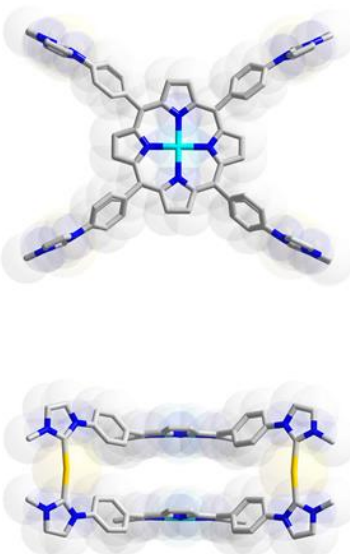

Zn---Zn $5.53 \AA$ b.
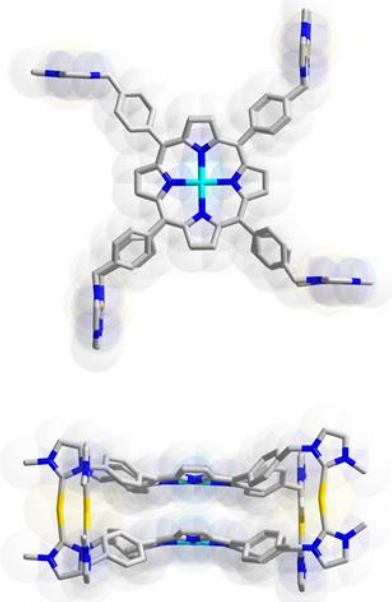

Zn---Zn $3.82 \AA$ c.
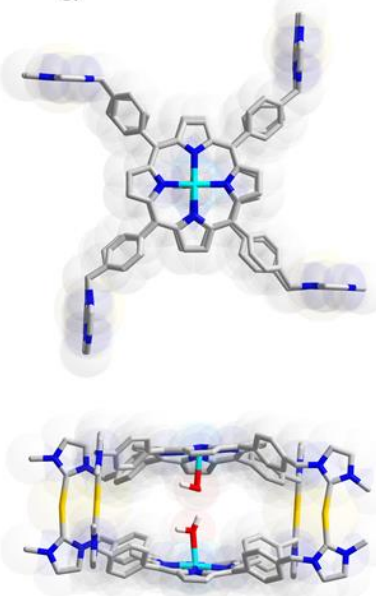

Zn---Zn $7.44 \AA$ d.
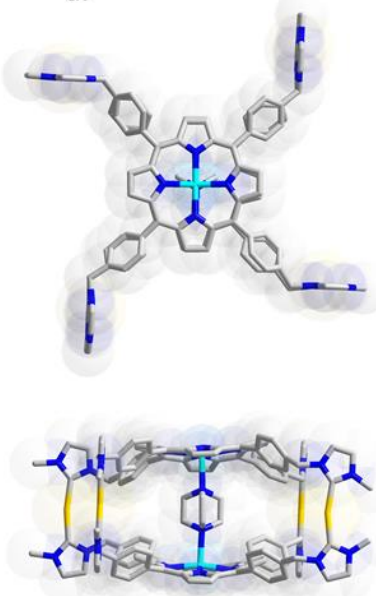

Zn---Zn $7.18 \AA$

Figure 5. DFT optimized geometries of empty porphyrin cages $\left[\mathbf{A u}_{4}(\mathbf{1} \mathbf{a} / \mathbf{b})_{2}\right]^{4+}$ (a), empty porphyrin cages $\left[\mathbf{A u}_{4}(\mathbf{1} \mathbf{c} / \mathbf{d})_{2}\right]^{4+}(b)$, porphyrin cages $\left[\mathbf{A u}_{4}(\mathbf{1} \mathbf{c} / \mathbf{d})_{2}\right]^{4+}$ encapsulating two $\mathrm{H}_{2} \mathrm{O}$ 
molecules (c), porphyrin cages $\left[\mathbf{A u}_{4}(\mathbf{1} \mathbf{c} / \mathbf{d})_{2}\right]^{4+}$ encapsulating DABCO (d). Alkyl chains were replaced by methyl groups to shorten computing time. $\mathrm{H}$ atoms are omitted for clarity, except those of $\mathrm{H}_{2} \mathrm{O}$ molecules. See the Supplementary Information for computational details.

Following these observations, we turned our attention to the bifunctional ligand 1,4diazabicyclo[2.2.2]-octane (DABCO), a typical guest used as probe to investigate encapsulation properties of cofacial porphyrin dimers ${ }^{63}$ or as template for their synthesis. ${ }^{64-66}$ First, we investigated the encapsulation properties of the rigid porphyrin cage $\left[\mathbf{A u}_{4}\left(\mathbf{1 a}_{2}\right)_{2}\right]\left(\mathbf{P F}_{6}\right)_{4}$ (Figure 6ac). One equivalent of DABCO was added to a solution of the porphyrin cage $\left[\mathbf{A u}_{\mathbf{4}}\left(\mathbf{1 a}_{\mathbf{2}}\right)_{2}\right]\left(\mathbf{P F}_{\mathbf{6}}\right)_{4}$ in $\mathrm{CD}_{3} \mathrm{CN}$ at $25{ }^{\circ} \mathrm{C}$ and a weak upfield singlet was observed by ${ }^{1} \mathrm{H}$ NMR spectroscopy at $\delta=-5.21$ ppm (Figure 6b, red arrow). This signal is attributed to the protons of DABCO sandwiched between two porphyrins (free $\mathrm{DABCO}$ in $\mathrm{CD}_{3} \mathrm{CN}, \delta=2.65 \mathrm{ppm}$ ). However, it may correspond to two possible structures (Figure 7), i.e. inclusion complexes (coordination of DABCO inside the porphyrin cage) or ternary complexes (coordination of DABCO outside bridging two porphyrin cages). To answer this question, the $\mathrm{DABCO} /\left[\mathrm{Au}_{4}\left(\mathbf{1 a}_{2}\right)_{2}\right]\left(\mathbf{P F}_{6}\right)_{4}$ ratio was increased up to 5 and we observed that the weak upfield signal due to DABCO disappeared (Figure 6c). This is due to the decomposition of ternary complexes upon coordination of additional DABCO as it was previously observed by Sanders and coworkers with analogous systems. ${ }^{63}$ No additional upfield singlet could be observed by ${ }^{1} \mathrm{H}$ NMR spectroscopy by reacting the porphyrin cage $\left[\mathbf{A u}_{4}\left(\mathbf{1 a}_{2}\right)_{2}\right]\left(\mathbf{P F}_{6}\right)_{4}$ with DABCO. The singlets due to $\beta$-pyrrolic protons remain in the same range ( $\delta=8.55-8.58 \mathrm{ppm})$ upon addition of DABCO. This shows that the porphyrin-porphyrin distance is not modified and that there is no encapsulation of $\mathrm{DABCO}$ even after several hours. The rigidity of the porphyrin cage $\left[\mathbf{A u}_{4}\left(\mathbf{1}_{\mathbf{a}}\right)_{2}\right]\left(\mathbf{P F}_{6}\right)_{4}$ and the short porphyrin-porphyrin distance $(\sim 5.5 \AA$ according to DFT calculations) can explain this result. 

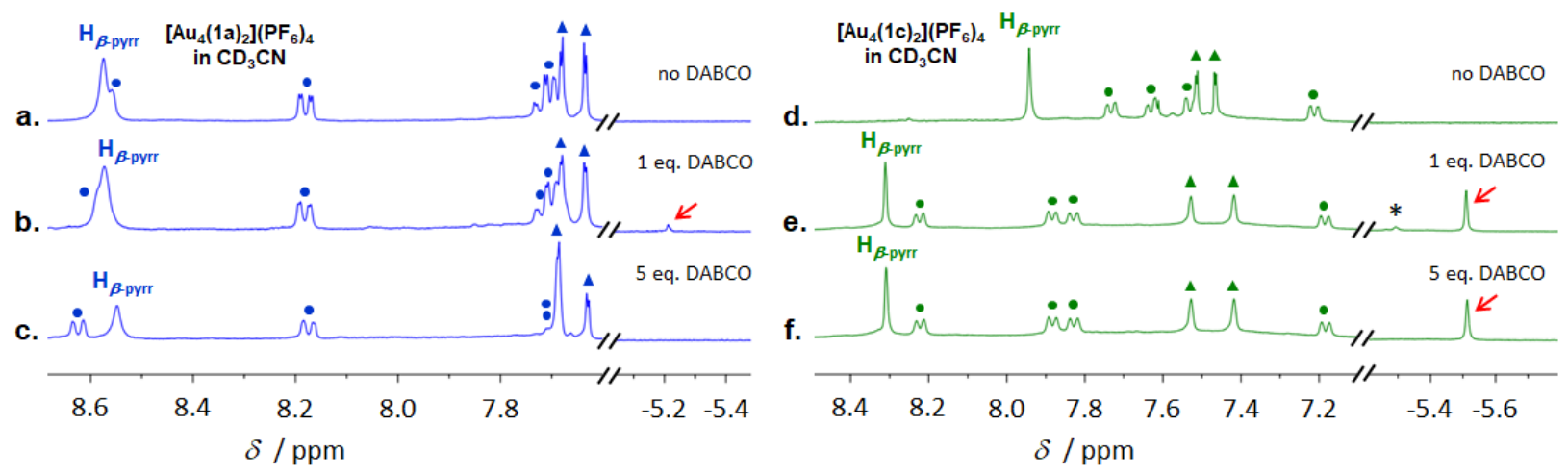

Figure 6. ${ }^{1} \mathrm{H}$ NMR spectra $(400 \mathrm{MHz})$ in $\mathrm{CD}_{3} \mathrm{CN}$ of the porphyrin cages $\left[\mathbf{A u}_{4}\left(\mathbf{1 a}_{2}\right)_{2}\right]\left(\mathbf{P F}_{\mathbf{6}}\right)_{4}$ (a) and $\left[\mathbf{A u}_{4}(\mathbf{1 c})_{2}\right]\left(\mathbf{P F}_{6}\right)_{4}(\mathrm{~b}) ; 15$ minutes after the addition of 1 equivalent of DABCO (b and e); 15 minutes after the addition of 5 equivalents of $\mathrm{DABCO}(\mathrm{c}$ and $\mathrm{f})(\bullet=$ signals of $m e s o$ aryl protons ; $\boldsymbol{\Delta}=$ signals of $\mathrm{H}^{4}$ and $\left.\mathrm{H}^{5}\right) . *$ The weak signal at $\delta=-5.29 \mathrm{ppm}$ in spectrum (e) may be due to the formation of trace amount of ternary complexes (coordination of DABCO outside bridging two porphyrin cages).

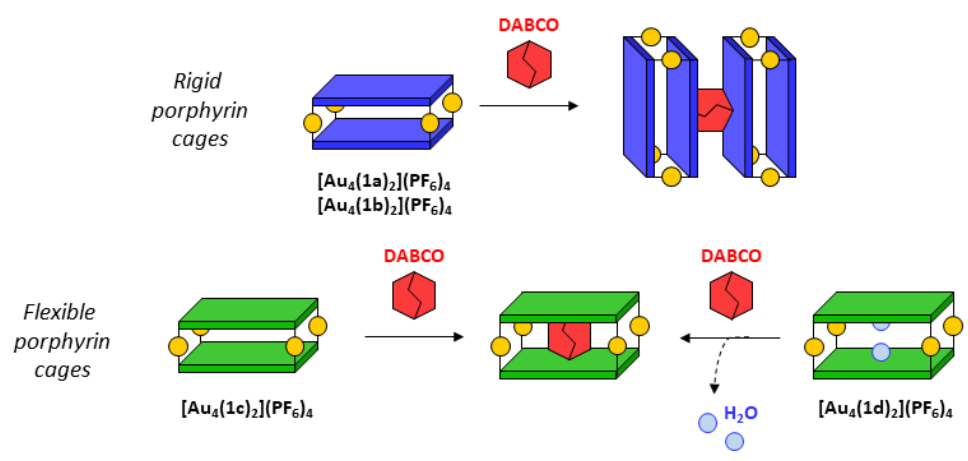

Figure 7. Schematic representation of the reactions of porphyrin cages with DABCO. 
Then, we investigated the encapsulation of DABCO by the more flexible porphyrin cages $\left[\mathbf{A u}_{4}(\mathbf{1 c})_{2}\right]\left(\mathbf{P F}_{6}\right)_{4}$ ("closed" conformation) and $\left[\mathbf{A u}_{4}\left(\mathbf{1 d}_{2}\right]\left(\mathbf{P F}_{6}\right)_{4}\right.$ ("open" conformation with encapsulated $\mathrm{H}_{2} \mathrm{O}$ molecules). DFT calculations show that flexible porphyrin cages $\left[\mathrm{Au}_{4}(\mathbf{1} \mathbf{c} / \mathbf{d})_{2}\right]^{4+}$ are able to encapsulate DABCO with a calculated $\mathrm{Zn}-\mathrm{Zn}$ distance of $7.14 \AA$ and structural features including porphyrin cores distortion similar to those calculated for the previous system with encapsulated $\mathrm{H}_{2} \mathrm{O}$ molecules (Figure $5 \mathrm{~d}$ ). The porphyrin cage [ $\left.\mathbf{A u}_{4}(\mathbf{1} \mathbf{c})_{2}\right]\left(\mathbf{P F}_{6}\right)_{4}$ was mixed with one equivalent of $\mathrm{DABCO}$ in $\mathrm{CD}_{3} \mathrm{CN}$ at $25{ }^{\circ} \mathrm{C}$ and a new set of signals was observed within minutes in the ${ }^{1} \mathrm{H}$ NMR spectrum of the reaction mixture (Figure 6e). The singlet due to the $\beta$ pyrrolic protons is significantly shifted downfield $(\Delta \delta=0.41 \mathrm{ppm})$ upon inclusion of DABCO suggesting a greater porphyrin-porphyrin distance in good agreement with DFT optimized geometries. An upfield signal integrating for $12 \mathrm{H}$ is observed at $\delta=-5.51 \mathrm{ppm}$ and corresponds to the signal of the protons of DABCO sandwiched between the two porphyrins of the cage (Figure 6e, red arrow). This complex is stable and did not decompose when the DABCO/[Au4 $\left.\left(\mathbf{1}_{\mathbf{c}}\right)_{2}\right]\left(\mathbf{P F}_{\mathbf{6}}\right)_{4}$ ratio was increased up to 5 confirming the formation of the inclusion complex $\left[\mathbf{D A B C O} @ \mathbf{A u}_{4}(\mathbf{1 c})_{2}\right]^{4+}$ (Figure 6f). The $D_{4 h}$ symmetry of the porphyrin cage $\left[\mathbf{A u}_{4}(\mathbf{1 c})_{2}\right]^{4+}$ is retained after inclusion of $\mathrm{DABCO}$ with $D_{3 \mathrm{~h}}$ symmetry. It is in agreement with a fast rotation of DABCO inside the porphyrin cage along the $\mathrm{Zn}---\mathrm{Zn}$ axis.

The same upfield signal of $\mathrm{DABCO}$ at $\delta=-5.51 \mathrm{ppm}$ and identical signals in the aromatic region were observed by ${ }^{1} \mathrm{H}$ NMR spectroscopy when the porphyrin cage $\left[\mathbf{A u}_{\mathbf{4}}\left(\mathbf{1 d}_{\mathbf{2}} \mathbf{2}_{\mathbf{2}}\left(\mathbf{P F}_{\mathbf{6}}\right]_{4}\right.\right.$ was mixed with one equivalent of $\mathrm{DABCO}$ in $\mathrm{CD}_{3} \mathrm{CN}$ at $25^{\circ} \mathrm{C}$ (Figure 8a). Here, the appearance of this singlet at $\delta=-5.51 \mathrm{ppm}$ is concomitant with the disappearance of the signal at $\delta=-6.27 \mathrm{ppm}$ due to encapsulated $\mathrm{H}_{2} \mathrm{O}$ molecules suggesting the occurrence of a guest exchange reaction. Compared to the previous system, the inclusion of DABCO in the porphyrin cage $\left[\mathbf{A u}_{4}\left(\mathbf{1 d}_{\mathbf{2}}\right)_{\mathbf{2}}\right]\left(\mathbf{P F}_{\mathbf{6}}\right)_{4}$ 
is significantly slower because signals of the remaining starting material could be observed after 4 hours (Figure 8a, middle): ${ }^{1} \mathrm{H}$ NMR signals of both porphyrin cages $\left[\mathbf{A} \mathbf{u}_{4}(\mathbf{1 d})_{2}\right]^{4+}$ including $\mathrm{H}_{2} \mathrm{O}$ molecules (green dots) and DABCO (red dots) were observed independently. After 16 hours, only one species corresponding to the inclusion complex [DABCO@Au4(1d) $\left.{ }_{2}\right]^{4+}$ was observed in solution (Figure 8a, bottom). DOSY ${ }^{1} \mathrm{H}$ NMR experiment shows that the porphyrin cage $\left[\mathrm{Au}_{4}(\mathbf{1 d})_{2}\right]^{4+}$ and DABCO diffuse at the same rate, which provides strong evidence that DABCO is encapsulated (Figure S129). 2D ${ }^{1} \mathrm{H}$ ROESY NMR spectrum showed a cross-peak of DABCO protons with meso aryl protons pointing inside the cavity, namely $\mathrm{H}_{o}$ in, confirming the encapsulation of DABCO between the two porphyrins (Figure S130). High-resolution ESI-TOF $(+$ ) mass spectrometry also supports the encapsulation of DABCO by the presence of a peak at $\mathrm{m} / \mathrm{z}=$ 836.2723 Da, which could be assigned to the tetracationic species $\left[\mathbf{D A B C O} @ \mathbf{A u}_{4}(\mathbf{1 d})_{2}\right]^{4+}($ Figure 8b). Finally, binding of DABCO was investigated by UV-visible absorption spectroscopy. Addition of DABCO leads to a slight redshift of the Soret absorption band from 421 to $423 \mathrm{~nm}$. UV-Visible titration experiment with 0-3 equivalents of DABCO shows the presence of isobestic points indicating that only two species exist in solution: the starting material $\left[\mathbf{A u}_{4}(\mathbf{1 d})_{2}\right]^{4+}$ containing $\mathrm{H}_{2} \mathrm{O}$ molecules and the inclusion complex $\left[\mathbf{D A B C O} @ \mathbf{A u}_{4}(\mathbf{1 d})_{2}\right]^{4+}$ (Figure 8c). Satisfyingly, this is in agreement with NMR studies (Figure 8a). The absence of spectral evolution with DABCO $>1$ equivalent confirms that the inclusion complex $\left[\mathbf{D A B C O} @ \mathbf{A u} \mathbf{4}(\mathbf{1 d})_{2}\right]^{4+}$ persists in solution even in the presence of an excess of DABCO. A binding constant of $K_{\mathrm{a}}=1.3 \times 10^{5} \mathrm{M}^{-}$ ${ }^{1}$ was calculated using a Benesi-Hildebrand plot. Coordination of a second DABCO was not observed. 


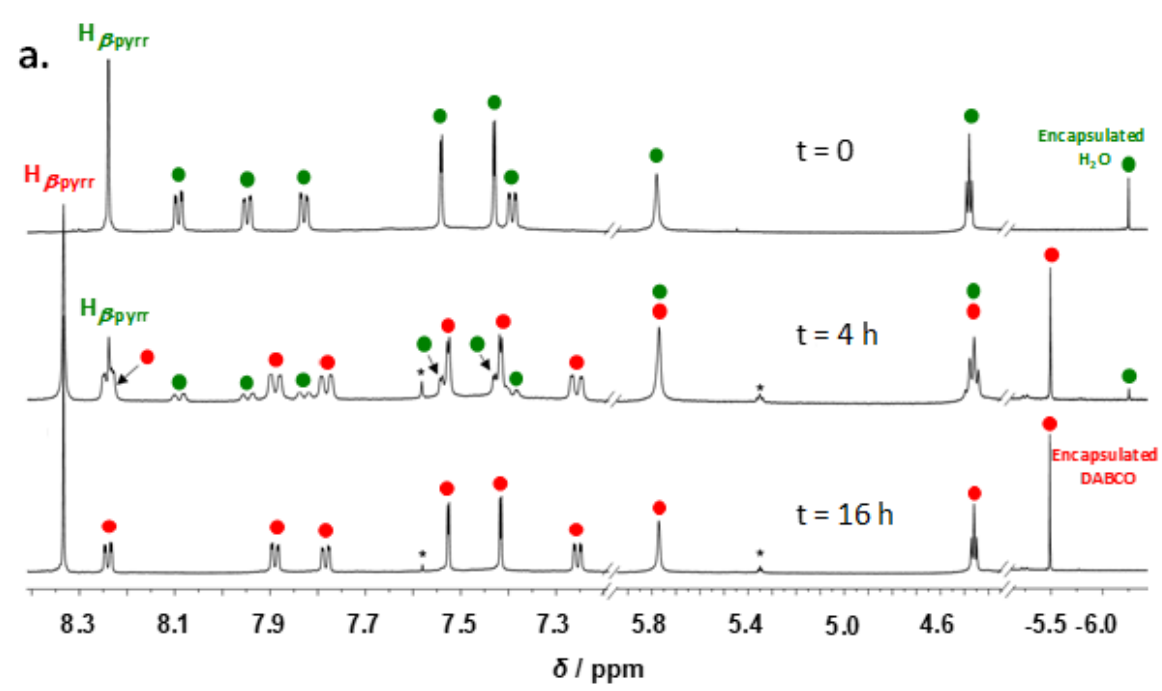

b.

c.
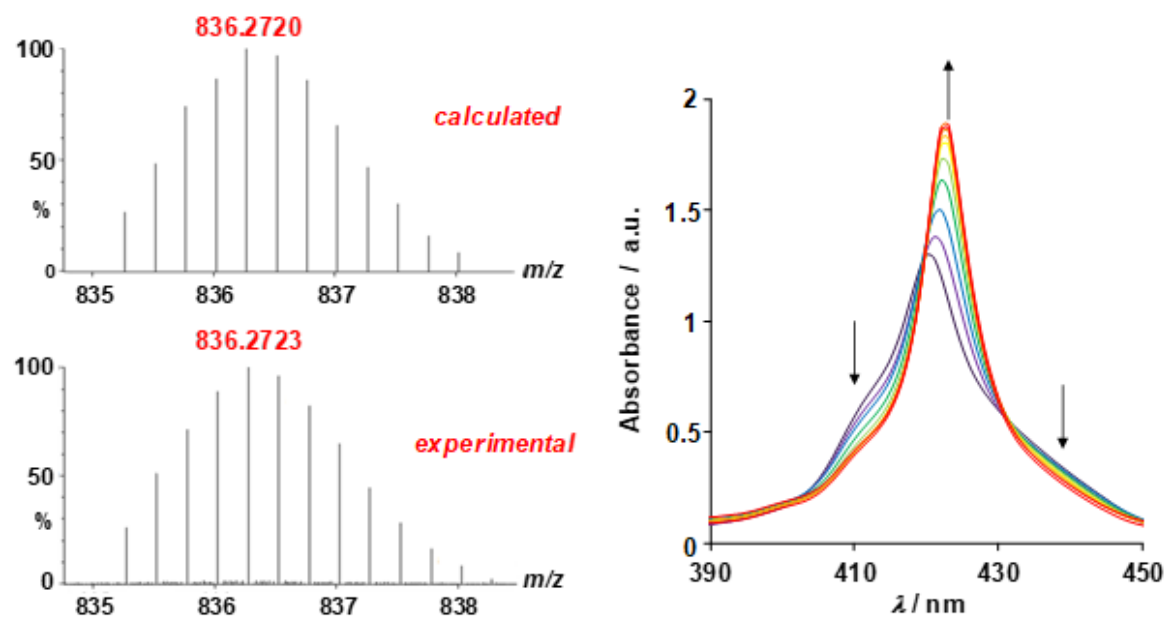

Figure 8. (a) ${ }^{1} \mathrm{H}$ NMR spectra of porphyrin cage $\left[\mathbf{A u}_{4}(\mathbf{1 d})_{2}\right]\left(\mathbf{P F}_{6}\right)_{4}$ in $\mathrm{CD}_{3} \mathrm{CN}$ (top), 4 hours after the addition of 1 equivalent of DABCO (middle), 16 hours after the addition of 1 equivalent of DABCO (bottom); spectra at $\mathrm{t}=0$ and $\mathrm{t}=16$ hours were recorded at $600 \mathrm{MHz}$; spectrum at $\mathrm{t}=4$ hours was recorded at $400 \mathrm{MHz}$; signals marked with * = impurities; (b) HR ESI(+) mass spectrum of $\left[\mathbf{A u}_{4}(\mathbf{1 d})_{2}\right]\left(\mathbf{P F}_{6}\right)_{4}$ encapsulating DABCO: experimental (bottom) and calculated (top) isotopic distribution profiles of the tetracationic species $\mathrm{C}_{158} \mathrm{H}_{164} \mathrm{~N}_{26} \mathrm{Zn}_{2} \mathrm{Au}_{4}{ }^{4+}$, namely [DABCO@Au4(1d)2 ${ }^{4+}$; (c) UV-Visible absorption spectra of $\left[\mathbf{A u}_{4}(\mathbf{1 d})_{2}\right]\left(\mathbf{P F}_{6}\right)_{4}(2.5 \mu \mathrm{M}$ in $\left.\mathrm{CH}_{3} \mathrm{CN}\right) 24$ hours after addition of $\mathrm{DABCO}(0,0.2,0.4,0.6,0.8,1,1.2,1.5,2$ and 3 equivalents). 


\section{CONCLUSION}

In summary, four porphyrins equipped with imidazolium rings on the para positions of the meso aryl groups were prepared and used as NHC precursors for the synthesis of porphyrin cages assembled from eight metal-carbene bonds. The reversibility of the $\mathrm{Ag}^{+}-\mathrm{C}_{\mathrm{NHC}}$ bonds was used to our advantage to allow two porphyrins to self-assemble with a face-to-face orientation and to further transmetalate $\mathrm{NHC}$ ligands from $\mathrm{Ag}(\mathrm{I})$ to $\mathrm{Au}(\mathrm{I})$. In solution, we found that the conformation and the encapsulation properties of these cage-like architectures strongly depend on the structure of the $-\left(\mathrm{CH}_{2}\right)_{\mathrm{n}}-\mathrm{NHC}$ side arms on the para positions of the meso aryl groups. Indeed, in absence of methylene functions $(n=0)$, the two porphyrins are in close proximity and the obtained rigid systems are not able to act as host to encapsulate a typical guest like DABCO. In this case, external coordination of DABCO on zinc(II) porphyrins was observed. By contrast, the presence of methylene functions $(\mathrm{n}=1)$ between meso aryl groups and peripheral NHCs bring additional flexibility to the systems allowing the inner space between the two porphyrins to expand enough to encapsulate $\mathrm{H}_{2} \mathrm{O}$ molecules or DABCO. More surprisingly, the length of the peripheral alkyl chains used as NHC-wingtip groups (n-hexyl vs. $n$-butyl) also dramatically modifies the conformation of the flexible porphyrin cages in solution and their encapsulating properties. To conclude, we demonstrate here that host-guest chemistry is feasible with porphyrin cages built upon formation of NHC-metal bonds and encapsulation of other guests may be considered in the future. The possibility to use porphyrin cages assembled from NHC-metal bonds for catalysis is also currently under investigation in our group. 


\section{ASSOCIATED CONTENT}

\section{Supporting Information.}

The Supporting Information is available free of charge at https://pubs.acs.org/. Additional experimental details and experimental procedures for the preparation of porphyrins 4a-d, NMR spectra, mass spectra and UV-visible absorption spectra for all compounds.

\section{AUTHOR INFORMATION}

\section{Corresponding Author}

Sébastien Richeter - ICGM, Univ Montpellier, CNRS, ENSCM, Montpellier 34293, France; Email: sebastien.richeter@umontpellier.fr

\section{Authors}

Ludivine Poyac - ICGM, Univ Montpellier, CNRS, ENSCM, Montpellier 34293, France

Clémence Rose - ICGM, Univ Montpellier, CNRS, ENSCM, Montpellier 34293, France

Mohammad Wahiduzzaman - ICGM, Univ Montpellier, CNRS, ENSCM, Montpellier 34293, France

Aurélien Lebrun - LMP, Université de Montpellier, Montpellier 34293, France

Guillaume Cazals - LMP, Université de Montpellier, Montpellier 34293, France

Charles H. Devillers - ICMUB UMR 6302, CNRS, Univ Bourgogne Franche-Comté, 9 avenue Alain Savary, Dijon 21078, France

Pascal G. Yot - ICGM, Univ Montpellier, CNRS, ENSCM, Montpellier 34293, France 
Sébastien Clément - ICGM, Univ Montpellier, CNRS, ENSCM, Montpellier 34293, France

\section{Author Contributions}

\| L. P. and C. R. contributed equally to this work. The manuscript was written through contributions of all authors. All authors have given approval to the final version of the manuscript.

\section{ACKNOWLEDGMENT}

The authors are grateful to the French Agence Nationale de la Recherche (ANR-19-CE070009-01 and ANR-15-CE29-0018-01), the University of Montpellier and the Centre National de la Recherche Scientifique (CNRS) for financial support.

\section{REFERENCES}

(1) Northrop, B. H.; Zheng, Y.-R.; Chi, K.-W.; Stang, P. J. Self-Organization in Coordination-Driven Self-Assembly. Acc. Chem. Res. 2009, 42, 1554-1563.

(2) Chakrabarty, R.; Mukherjee, P. S.; Stang, P. J. Supramolecular Coordination: SelfAssembly of Finite Two- and Three-Dimensional Ensembles. Chem. Rev. 2011, 111, 6810-6918.

(3) Krämer, R.; Lehn, J.-M.; Marquis-Rigault, A. Self-recognition in helicate selfassembly: spontaneous formation of helical metal complexes from mixtures of ligands and metal ions. Proc. Natl. Acad. Sci. USA 1993, 90, 5394-5398.

(4) Caulder, D. L., Raymond, K. N. Superamolecular Self-Recognition and Self-Assembly in Gallium(III) Catecholamide Triple Helices. Angew. Chem. Int. Ed. 1997, 36, 14401442.

(5) Dietrich-Buchecker, C. O.; Sauvage, J.-P.; Armaroli, N.; Ceroni, P.; Balzani, V. Knotted Heterodinuclear Complexes. Angew. Chem. Int. Ed. 1996, 35, 1119-1121. 
(6) Meyer, C. D.; Forgan, R. S.; Chichak, K. S.; Peters, A. J.; Tangchaivang, N.; Cave, G. W. V.; Khan, S. I.; Cantrill, S. J.; Stoddart, J. F. The Dynamic Chemistry of Molecular Borromean Rings and Solomon Knots. Chem. - Eur. J. 2010, 16, 12570-12581.

(7) Domoto, Y.; Abe, M.; Kikuchi, T.; Fujita, M. Self-Assembly of Coordination Polyhedra with Highly Entangled Faces Induced by Metal-Acetylene Interactions. Angew. Chem. Int. Ed. 2020, 59, 3450-3454.

(8) Fujita, M.; Tominaga, M.; Hori, A.; Therrien, B. Coordination Assemblies from a Pd(II)-Cornered Square Complex. Acc. Chem. Res. 2005, 38, 371-380.

(9) Fulong, C. R. P.; Guardian, M. G. E.; Aga, D. S.; Cook. T. R. A Self-Assembled Iron(II) Metallacage as a Trap for Per- and Polyfluoroalkyl Substances in Water. Inorg. Chem. 2020, 59, 6697-6708.

(10) Shanmugaraju, S.; Mukherjee, P. S. Self-Assembled Discrete Molecules for Sensing Nitroaromatics. Chem. Eur. J. 2015, 21, 6656-6666.

(11) Pluth, M. D.; Bergman, R. G.; Raymond, K. N. Acid Catalysis in Basic Solution: A Supramolecular Host Promotes Orthoformate Hydrolysis. Science 2007, 316, 85-88.

(12) Kaphan, D. M.; Toste, F. D.; Bergman, R. G.; Raymond, K. N. Enabling New Modes of Reactivity via Constrictive Binding in a Supramolecular-Assembly-Catalyzed AzaPrins Cyclization. J. Am. Chem. Soc. 2015, 137, 9202-9205.

(13) Gao, W.-X.; Zhang, H.-N.; Jin, G.-X. Supramolecular catalysis based on discrete heterometallic coordination-driven metallacycles and metallacages. Coord. Chem. Rev. 2019, 386, 69-84.

(14) Yoshizawa, M.; Tamura, M.; Fujita, M. Diels-Alder in Aqueous Molecular Hosts: Unusual Regioselectivity and Efficient Catalysis. Science 2006, 312, 251-254.

(15) Yoshizawa, M.; Klosterman, J. K.; Fujita, M. Functional Molecular Flasks: New Properties and Reactions within Discrete, Self-Assembled Hosts. Angew. Chem., Int. Ed. 2009, 48, 3418-3438. 
(16) Yu, G.; Shan; S.; Manik, L.; Zhou, J.; Cook, T. R.; Yung, B. C.; Chen, J.; Mao, Z.; Zhang, F.; Zhou, Z.; Liu, Y.; Shao, L.; Wang, S.; Gao, C.; Huang, F.; Stang, P. J.; Chen, $\mathrm{X}$. A discrete organoplatinum(II) metallacage as a multimodality theranostic platform for cancer photochemotherapy. Nat. Commun. 2018, 9, 1-18.

(17) Sepehrpour, H.; Fu, W.; Sun, Y.; Stang, P. J. Biomedically Relevant Self-Assembled Metallacycles and Metallacages. J. Am. Chem. Soc. 2019, 14005-14020.

(18) Casini, A.; Woods, B.; Wenzel, M. The Promise of Self-Assembled 3D Supramolecular Coordination Complexes for Biomedical Applications. Inorg. Chem. 2017, 56, 1471514729.

(19) Cook, T. R.; Vajpayee, V.; Lee, M. H.; Stang, P. J.; Chi, K.-W. Biomedical and Biochemical Applications of Self-Assembled Metallacycles and Metallacages. Acc. Chem. Res. 2013, 46, 2464-2474.

(20) Durot, S.; Taesch, J.; Heitz, V. Multiporphyrinic Cages: Architectures and Functions. Chem. Rev. 2014, 114, 8542-8578.

(21) Garcia-Simon, C.; Monferrer, A.; Garcia-Borras, M.; Imaz, I.; Maspoch, D.; Costas, M.; Ribas, X. Size-selective encapsulation of $\mathrm{C}_{60}$ and $\mathrm{C}_{60}$-derivatives within an adaptable naphthalene-based tetragonal prismatic supramolecular nanocapsule. Chem. Commun. 2019, 55, 798-801.

(22) Fuertes-Espinosa, C.; Gomez-Torres, A.; Morales-Martinez, R.; Rodriguez-Fortea, A.; Garcia-Simon, C.; Gandara, F.; Imaz, I.; Juanhuix, J.; Maspoch, D.; Poblet, J. M.; Echegoyen, L.; Ribas, X. Angew. Chem. Int. Ed. 2018, 57, 11294-11299.

(23) S. P. Black, D. M. Wood, F. B. Schwarz, T. K. Ronson, J. J. Holstein, A. R. Stefankiewicz, C. A. Schalley, J. K. M. Sanders, J. R. Nitschke. Catenation and encapsulation induce distinct reconstitutions within a dynamic library of mixed-ligand $\mathrm{Zn}_{4} \mathrm{~L}_{6}$ cages. Chem. Sci. 2016, 7, 2614-2620. 
(24) Nakamura, T.; Ube, H.; Miyake, R.; Shionoya, M. A C60-Templated Tetrameric Porphyrin Barrel Complex via Zinc-Mediated Self-Assembly Utilizing Labile Capping Ligands J. Am. Chem. Soc. 2013, 135, 18790-18793.

(25) Meng, W.; Breiner, B.; Rissanen, K.; Thoburn, J. D.; Clegg, J. K.; Nitschke, J. R. A Self-Assembled $\mathrm{M}_{8} \mathrm{~L}_{6}$ Cubic Cage that Selectively Encapsulates Large Aromatic Guests. Angew. Chem. Int. Ed. 2011, 50, 3479-3483.

(26) Wang, X.; Nurttila, S. S.; Dzik, W.; Becker, R.; Rodgers, J.; Reek, J. N. H. Tuning the Porphyrin Building Block in Self-Assembled Cages for Branched-Selective Hydroformylation of Propene. Chem. Eur. J. 2017, 23, 14769-14777.

(27) Garcia-Simon, C.; Gramage-Doria, R.; Raoufmoghaddam, S.; Parella, T.; Costas, M.; Ribas, X.; Reek, J. N. H. Enantioselective Hydroformylation by a Rh-Catalyst Entrapped in a Supramolecular Metallocage. J. Am. Chem. Soc. 2015, 137, 2680-2687.

(28) Oliveri, G.; Gianneschi, N. C.; Nguyen, S. T.; Mirkin, C. A.; Stern, C. L.; Wawrzak, Z.; Pink, M. Supramolecular Allosteric Cofacial Porphyrin Complexes. J. Am. Chem. Soc. 2006, 128, 16286-16296.

(29) Hiroto, S.; Miyake, Y.; Shinokubo, H. Synthesis and Functionalization of Porphyrins through Organometallic Methodologies Chem. Rev. 2017, 117, 2910-3043.

(30) Aratani, N.; Kim, D.; Osuka, A. Discrete Cyclic Porphyrin Arrays as Artificial LightHarvesting Antenna Acc. Chem. Res. 2009, 42, 1922-1934.

(31) Camus, J.-M.; Aly, S. M.; Stern, C.; Guilard, R.; Harvey, P. D. Acceleration of the through space $\mathrm{S} 1$ energy transfer rates in cofacial bisporphyrin bio-inspired models by virtue of substituents effect on the Förster $J$ integral and its implication in the antenna effect in the photosystems. Chem. Commun. 2011, 47, 8817-8819.

(32) Gros, C. P.; F. Brisach, F.; Meristoudi, A.; Espinosa, E.; Guilard, G.; Harvey, P. D. Modulation of the singlet-singlet through-space energy transfer rates in cofacial bisporphyrin and porphyrin-corrole dyads. Inorg. Chem. 2007, 46, 125-135. 
(33) Tanaka, M.; Ohkubo, K.; Gros, C. P.; Guilard, R.; Fukuzumi, S. Persistent ElectronTransfer State of a $\pi$-Complex of Acridinium Ion Inserted between Porphyrin Rings of Cofacial Bisporphyrins. J. Am. Chem. Soc. 2006, 128, 14625-14633.

(34) Mondal, P.; Rath, S.P. Cyclic metalloporphyrin dimers: Conformational flexibility, applications and future prospects. Chem. Soc. Rev. 2020, 405, 213117.

(35) A.L. Kieran, S. I. Pascu, T. Jarrosson, J. K. M. Sanders, Inclusion of $\mathrm{C}_{60}$ into an adjustable porphyrin dimer generated by dynamic disulfide chemistry. Chem. Commun. 2005, 1276-1278.

(36) Nakamura, T.; Ube, H.; Shionoya, M. Silver-Mediated Formation of a Cofacial Porphyrin Dimer with the Ability to Intercalate Aromatic Molecules. Angew. Chem. Int. Ed., 2013, 52, 12096-12100.

(37) R. R. Durand, Jr, R. R.; Bencosme, C. S.; Collman, J. P.; Anson, F. C. Mechanistic aspects of the catalytic reduction of dioxygen by cofacial metalloporphyrins. J. Am. Chem. Soc. 1983, 105, 2710-2718.

(38) Fukuzumi, S.; Okamoto, K.; Gros, C. P.; Guilard, R. Mechanism of Four-Electron Reduction of Dioxygen to Water by Ferrocene Derivatives in the Presence of Perchloric Acid in Benzonitrile, Catalyzed by Cofacial Dicobalt Porphyrins. J. Am. Chem. Soc. 2004, 126, 10441-10449.

(39) Mohamed, E. A.; Zahran, Z. N.; Naruta, Y. Efficient electrocatalytic CO2 reduction with a molecular cofacial iron porphyrin dimer. Chem. Commun. 2015, 51, 1690016903.

(40) Oldacre, A. N.; Friedman, A. E.; Cook, T. R. A Self-Assembled Cofacial Cobalt Porphyrin Prism for Oxygen Reduction Catalysis. J. Am. Chem. Soc. 2017, 139, 14241427.

(41) Oldacre, A. N.; Crawley, M. R.; Friedman, A. E.; Cook, T. R. Tuning the Activity of Heterogeneous Cofacial Cobalt Porphyrins for Oxygen Reduction Electrocatalysis through Self-Assembly. Chem. Eur. J. 2018, 24, 10984-10987. 
(42) Rose, C.; Lebrun, A.; Clément, S.; Richeter, S. Cofacial porphyrin dimers assembled from N-heterocyclic carbene-metal bonds. Chem. Commun. 2018, 54, 9603-9606.

(43) Usón, R.; Laguna, A., Laguna, M. (Tetrahydrothiophene)gold(I) or Gold(III) Complexes. Inorg Synth. 1986, 26, 85-91.

(44) Subbaiyan, N. K.; Maligaspe, E.; D’Souza, F. Near Unity Photon-to-Electron Conversion Efficiency of Photoelectrochemical Cells Built on Cationic Water-Soluble Porphyrins Electrostatically Decorated onto Thin-Film Nanocrystalline SnO2 Surface. ACS Appl. Mater. Interfaces 2011, 3, 2368-2376.

(45) Jin, R.-H.; Aoki, S.; Shima, K. A new route of water soluble porphyrins: phosphonium and ammonium type cationic porphyrins and self-assembly. Chem. Commun. 1996, 1939-1940.

(46) de Frémont, P.; Marion, N.; Nolan, S. P. Carbenes: Synthesis, properties, and organometallic chemistry. Coord. Chem. Rev. 2009, 253, 862-892.

(47) Herrmann, W. A.; Köcher, C. N-Heterocyclic Carbenes. Angew. Chem. Int. Ed. 1997, $36,2162-2187$

(48) Díez-González, S.; Marion, N.; Nolan, S. P. N-Heterocyclic Carbenes in Late Transition Metal Catalysis. Chem. Rev. 2009, 109, 3612-3676.

(49) Garrison, J. C.; Youngs, W. J. Ag(I) N-Heterocyclic Carbene Complexes: Synthesis, Structure, and Application. Chem. Rev. 2005, 105, 3978-4008.

(50) Ga, M.-M.; Liu, J.-Q.; Zhang, L.; Wang, Y.-Y.; Hahn, F. E.; Han, Y.-F. Preparation and Post-Assembly Modification of Metallosupramolecular Assemblies from Poly(NHeterocyclic Carbene) Ligands. Chem. Rev. 2018, 118, 9587-9641.

(51) Sinha, N.; Hahn, F. E. Metallosupramolecular Architectures Obtained from Poly-Nheterocyclic Carbene Ligands. Acc. Chem. Res. 2017, 50, 2167-2184. 
(52) Rit, A.; Pape, T.; Hahn, F. E. Self-Assembly of Molecular Cylinders from Polycarbene Ligands and $\mathrm{Ag}^{\mathrm{I}}$ or Au${ }^{\mathrm{I}}$. J. Am. Chem. Soc. 2010, 132, 13, 4572-4573.

(53) Segarra, C.; Guisado-Barrios, G.; Hahn, F. E.; Peris, E. Hexanuclear Cylinder-Shaped Assemblies of Silver and Gold from Benzene-Hexa-N-heterocyclic Carbenes. Organometallics 2014, 33, 5077-5080.

(54) Maiti, N. C.; Mazumdar, S.; Periasamy, N. J- and H-Aggregates of PorphyrinSurfactant Complexes: Time-Resolved Fluorescence and Other Spectroscopic Studies. J. Phys. Chem. B 1998, 102, 1528-1538.

(55) Zanetti-Polzi, L.; Amadei, A.; Djemili, R.; Durot, S.; Schoepff, L.; Heitz, V.; Ventura, B.; Daidone, I. Interpretation of Experimental Soret Bands of Porphyrins in Flexible Covalent Cages and in Their Related Ag(I) Fixed Complexes. J. Phys. Chem. C 2019, 123, 13094-13103.

(56) Wylie, R. S.; Levy, E. G.; Sanders, J. K. M. Unexpectedly selective ligand binding within the cavity of a cyclic metalloporphyrin dimer. Chem. Commun. 1997, 16111612.

(57) Djemili, R.; Kocher L.; Durot, S.; Peuronen, A.; Rissanen, K.; Heitz, V. Positive Allosteric Control of Guests Encapsulation by Metal Binding to Covalent Porphyrin Cages. Chem. Eur. J. 2019, 25, 1481-1487.

(58) Nakash, M.; Sanders, J. K. M. Structure-Activity Relationships in the Acceleration of a Hetero Diels-Alder Reaction by Metalloporphyrin Hosts. J. Org. Chem. 2000, 65, 7266-7271.

(59) Mondal, P.; Banerjee, S.; Rath, S. P.; Prasad, S. Controlling the Photophysics of Aromatic Guests Using a Cyclic Porphyrin Dimer: Synthesis, Structure, and Encapsulation-Mediated "ON-OFF" Switch. Eur. J. Inorg. Chem. 2019, 2019, 36293637. 
(60) Liu, W.; Lin, C.; Weber, J. A.; Stern, C. L.; Young, R. M.; Wasielewski, M. R.; Stoddart, J. F. Cyclophane-Sustained Ultrastable Porphyrins. J. Am. Chem. Soc. 2020, $142,8938-8945$.

(61) Li, A.; Xiong, S.; Zhou, W.; Zhai, H.; Liu, Y.; He, Q. Superphane: a new lantern-like receptor for encapsulation of a water dimer. Chem. Commun. 2021, 57, 4496-4499.

(62) Zhang, R.; Murata, M.; Aharen, T.; Wakamiya A.; Shimoaka, T.; Hasegawa, T.; Murata, Y. Synthesis of a distinct water dimer inside fullerene $\mathrm{C}_{70}$. Nat. Chem. 2016, $8,435-441$.

(63) Hunter, C. A.; Meah, M. N.; Sanders, J. K. M. Dabco-metalloporphyrin binding: ternary complexes, host-guest chemistry and the measurement of pi-pi interactions. $J$. Am. Chem. Soc. 1990, 112, 5773-5780.

(64) Taesch, J.; Heitz, V.; Topić, F.; Rissanen, K. Templated synthesis of a large and flexible covalent porphyrinic cage bearing orthogonal recognition sites. Chem. Commun. 2012, 48, 5118-5120.

(65) Kocher, L.; Durot, S.; Heitz, V. Control of the cavity size of flexible covalent cages by silver coordination to the peripheral binding sites. Chem. Commun. 2015, 51, 1318113184.

(66) Ding, H.; Meng, X.; Cui, X.; Yang, Y.; Zhou, T.; Wang, C.; Zeller, M.; Wang, C. Highly-efficient synthesis of covalent porphyrinic cages via DABCO-templated imine condensation reactions. Chem. Commun. 2014, 50, 11162-11164. 


\section{Entry for the Table of Contents}

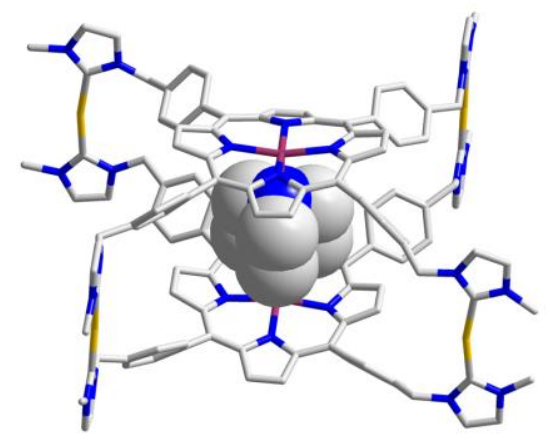

Porphyrin cages assembled from eight N-heterocyclic carbene-metal (NHC-M) bonds were synthesized and characterized $\left(\mathrm{M}=\mathrm{Ag}^{+}\right.$or $\left.\mathrm{Au}^{+}\right)$. Their conformation in solution and their encapsulation properties strongly depend on the nature of the linkers between porphyrins and NHCs. 